\title{
Evolution of asymptotic giant branch stars
}

\section{Optical to far-infrared isochrones with improved TP-AGB models}

\author{
P. Marigo ${ }^{1}$, L. Girardi ${ }^{2}$, A. Bressan ${ }^{2,3}$, M. A. T. Groenewegen ${ }^{4}$, L. Silva ${ }^{5}$, and G. L. Granato ${ }^{2}$ \\ 1 Dipartimento di Astronomia, Università di Padova, Vicolo dell’ Osservatorio 2, 35122 Padova, Italy \\ e-mail: paola.marigo@unipd.it \\ 2 Osservatorio Astronomico di Padova - INAF, Vicolo dell'Osservatorio 5, 35122 Padova, Italy \\ INAOE, Luis Enrique Erro 1, 72840 Tonantzintla, Puebla, Mexico \\ ${ }^{4}$ Instituut voor Sterrenkunde, Katholieke Universiteit Leuven, Celestijnenlaan 200 D, 3001 Leuven, Belgium \\ 5 Osservatorio Astronomico di Trieste - INAF, via Tiepolo 11, 34131 Trieste, Italy
}

Received 10 August 2007 / Accepted 29 January 2008

\section{ABSTRACT}

\begin{abstract}
We present a large set of theoretical isochrones, whose distinctive features mostly reside on the greatly-improved treatment of the thermally-pulsing asymptotic giant branch (TP-AGB) phase. Essentially, we have coupled the TP-AGB tracks described in Paper I, at their stages of pre-flash quiescent $\mathrm{H}$-shell burning, with the evolutionary tracks for the previous evolutionary phases from Girardi et al. (2000, A\&AS, 141, 371). Theoretical isochrones for any intermediate value of age and metallicity are then derived by interpolation in the grids. We take care that the isochrones keep, to a good level of detail, the several peculiarities present in these TP-AGB tracks e.g., the cool tails of C-type stars owing to the use of proper molecular opacities as convective dredge-up occurs along the TP-AGB; the bell-shaped sequences in the Hertzsprung-Russell (HR) diagram for stars with hot-bottom burning; the changes of pulsation mode between fundamental and first overtone; the sudden changes of mean mass-loss rates as the surface chemistry changes from M- to C-type; etc. Theoretical isochrones are then converted to about 20 different photometric systems - including traditional ground-based systems, and those of recent major wide-field surveys such as SDSS, OGLE, DENIS, 2MASS, UKIDSS, etc., - by means of synthetic photometry applied to an updated library of stellar spectra, suitably extended to include C-type stars. Finally, we correct the predicted photometry for the effect of circumstellar dust during the mass-losing stages of the AGB evolution, which allows us to improve the results for the optical-to-infrared systems, and to simulate mid- and far-IR systems such as those of Spitzer and AKARI. We illustrate the most striking properties of these isochrones by means of basic comparisons with observational data for the Milky Way disc and the Magellanic Clouds. Access to the data is provided both via a web repository of static tables (http://stev.oapd.inaf.it/ dustyAGB07 and CDS), and via an interactive web interface (http://stev.oapd.inaf.it/cmd), which provides tables for any intermediate value of age and metallicity, for several photometric systems, and for different choices of dust properties.
\end{abstract}

Key words. astronomical data bases: miscellaneous - stars: AGB and post-AGB - stars: carbon - stars: evolution galaxies: Magellanic Clouds - stars: Hertzsprung-Russell (HR) and C-M diagrams

\section{Introduction}

Theoretical isochrones find a wide range of applications in modern astrophysics, going from the simple age-dating of star clusters, to more complex problems like the derivation of star formation histories of resolved galaxies, and, since Charlot \& Bruzual (1991), the modelling of their spectrophotometric evolution across the Hubble time.

In fact, many different groups are publishing large grids of stellar isochrones, covering a wide enough range of stellar parameters (age, mass, metal content, alpha-enhancement, etc.) to become useful in the stellar population synthesis of galaxies. These isochrones normally comprehend a detailed description of all evolutionary phases, from the zero-age main sequence (ZAMS), up to either carbon ignition, or to the onset of the thermally-pulsing asymptotic giant branch phase (TP-AGB). The coverage of the TP-AGB evolution, however, has always been far from detailed and physically accurate, as we will illustrate below. The poor modelling of this phase greatly contrasts with its importance to the integrated light of stellar populations with ages from a few $10^{8} \mathrm{yr}$ up to the Hubble time.
According to Frogel et al. (1990), the TP-AGB contribution to the total luminosity of single-burst stellar populations reaches a maximum of about 40 percent at ages from 1 to $3 \mathrm{Gyr}$, falling to less than 10 percent at 10 Gyr. Obviously, TP-AGB stars have a great impact on the rest-frame near-infrared spectra of galaxies. Moreover, TP-AGB stars account for most of the bright-infrared (IR) objects in resolved galaxies, as clearly demonstrated by DENIS, 2MASS, SAGE and $\mathrm{S}^{3} \mathrm{MC}$ data for the Magellanic Clouds (Cioni et al. 1999; Nikolaev \& Weinberg 2000; Blum et al. 2006; Bolatto et al. 2007).

The situation regarding the TP-AGB phase in published sets of isochrones is the following: Whereas early isochrones sets do not even include the TP-AGB phase, more recent ones include it, but in a very approximate way, ignoring crucial aspects of the TP-AGB evolution. A summary table of available isochrones is provided in Marigo (2007) and updated in Table 1 herewith. In short, Bertelli et al. (1994) and Girardi et al. (2000, hereafter Gi00) adopt a very simple TP-AGB synthetic model that does not include the third dredge-up and the over-luminosity above the core mass-luminosity relation caused by hot bottom burning (HBB). A luminosity correction for stars undergoing HBB is 
Table 1. Available stellar isochrones including the TP-AGB phase.

\begin{tabular}{lcccl}
\hline \hline Reference & $3^{\text {rd }}$ DU and $\mathrm{HBB}^{a}$ & $T_{\mathrm{eff}^{b}}$ & Dust $^{c}$ & Public www address for retrieval $^{\circ}$ \\
\hline Bertelli et al. (1994) & NO & solar-scaled comp. & NO & http://pleiadi.pd.astro.it \\
Bressan et al. (1998) & NO & solar scaled comp. & YES & upon request \\
Girardi et al. (2000) & NO & solar-scaled comp. & NO & http://pleiadi.pd.astro.it \\
Marigo \& Girardi (2001) & YES & solar-scaled comp. & NO & http://pleiadi.pd.astro.it \\
Mouhcine (2002) & YES & solar scaled comp. & YES & upon request \\
Piovan et al. (2003) & NO & solar scaled comp. & YES & upon request \\
Marigo et al. (2003a) & YES & O-/C-rich comp. & NO & upon request \\
Cioni et al. (2006) & YES & O-/C-rich comp. & NO & http://pleiadi.pd.astro.it \\
Cordier et al. (2007) & NO & solar scaled comp. & NO & http://www.oa-teramo.inaf.it/BASTI \\
This work & YES & O-/C-rich comp. & YES & http://stev.oapd.inaf.it/cmd \\
\hline
\end{tabular}

\footnotetext{
${ }^{a}$ Explicit inclusion or not of AGB nucleosynthesis, i.e. the third dredge-up and hot-bottom burning, in the underlying AGB models.

${ }^{b}$ Choice of low-temperature opacities affecting the predicted effective temperature, i.e. for solar-scaled or variable $(\mathrm{O}-$ or $\mathrm{C}$-rich) chemical mixtures.

${ }^{c}$ Inclusion or not of circumstellar dust in the computation of the emitted spectrum.
}

instead included by Cordier et al. (2007), but in this case the third dredge-up - together with its most remarkable consequence, i.e. the formation of $\mathrm{C}$ stars - and the HBB nucleosynthesis are completely missing. Marigo \& Girardi (2001) present the first isochrones based on tracks (Marigo et al. 1999) with the third dredge-up included, and moreover, its efficiency is calibrated to reproduce the C-star luminosity functions in both Magellanic Clouds (MC) ${ }^{1}$. Another distinctive aspect of Marigo et al. (1999) tracks, is that the HBB efficiency, together with the model effective temperatures, are derived from numerical integrations of the envelope structure.

Besides the above mentioned works, the literature abounds in descriptions of evolutionary population synthesis models which have included the complete TP-AGB evolution (e.g. Charlot \& Bruzual 2003; Raimondo et al. 2005; Vázquez \& Leitherer 2005; only to mention a few). However, in all these cases, critical processes of TP-AGB evolution - again the third dredge-up, HBB, or superwind-like mass loss - are missing in the models. In some cases (e.g. Vázquez \& Leitherer 2005; Charlot \& Bruzual 2003), the tracks for the TP-AGB and previous evolutionary phases come from different sources; this implies that basic stellar properties like the core mass and envelope chemical composition are discontinuous along the composite tracks. As demonstrated by Marigo \& Girardi (2001), discontinuities in these quantities also produce an energetic inconsistency in the sense that they violate the strict coupling between stellar integrated light and chemical yields implied by the condition of energy conservation.

A few authors have computed isochrones aimed at modelling spectral evolution of galaxies, which consider the effect of circumstellar dust around AGB stars (Bressan et al. 1998; Mouhcine 2002; Piovan et al. 2003), but without improving the treatment of the underlying TP-AGB evolution, with respect to those already mentioned.

In all the previously-mentioned cases, moreover, the effect of variable opacities along the TP-AGB evolution is completely ignored: the basic stellar properties, and in particular, the effective temperature $T_{\text {eff }}$, are derived using opacities valid for scaledsolar compositions, which are highly inadequate to describe the chemically-evolved envelopes of TP-AGB stars. The proper consideration of variable opacities is now known to be crucial (see

\footnotetext{
1 Many of the improvements in Marigo et al. (1999) TP-AGB tracks were already introduced by Groenewegen \& de Jong (1993, 1994a-c), who computed extended sets of tracks and performed population synthesis of resolved AGB stellar populations. Groenewegen \& de Jong, however, do not provide theoretical isochrones from their tracks.
}

Marigo 2002; Marigo et al. 2003a), especially for stars undergoing the transition to the C-rich phase - i.e., comprising, for MC metallicities, all stars with initial masses between about 1.5 and $3.5 M_{\odot}$, and all isochrones with ages between about 0.5 and 5 Gyr. This situation is partially remedied by Cioni et al. (2006), who has made publicly available the first isochrone sets in which variable molecular opacities were considered along the TP-AGB evolution ${ }^{2}$. In fact, the C-star phase in these isochrones is characterized by much lower temperatures (reaching $T_{\text {eff }}$ as low as $2300 \mathrm{~K}$ ) than previously published isochrones. Those specific TP-AGB models, however, preceded the extensive calibration of dredge-up efficiency performed in Marigo \& Girardi (2007, hereafter Paper I). In this latter work, the dredge upparameters are tuned so that the models reproduce the observed C-star luminosity functions in the Magellanic Clouds, and the TP-AGB lifetimes of $\mathrm{M}$ and $\mathrm{C}$ stars in Magellanic Cloud star clusters (Girardi \& Marigo 2007a).

The goal of the present paper is to remedy this highly unsatisfactory situation, providing the first set of isochrones in which the TP-AGB evolution is treated with a satisfactory level of completeness and detail - i.e. considering the crucial effects of third dredge-up, hot bottom burning, and variable molecular opacities - and calibrated at the same time, i.e., able to reproduce the basic observables of AGB stars in the MCs. Moreover, we wish to provide these results in many photometric systems and including the reprocessing of radiation by circumstellar dust in mass-losing stars, which is crucial for modelling of infrared photometry.

The properties of our stellar evolutionary tracks are briefly recalled in Sect. 2, which discusses the basic aspects of constructing isochrones with the new TP-AGB tracks of Paper I including new variables like the $\mathrm{C} / \mathrm{O}$ ratio, pulsation periods, pulsation modes, etc. Section 3 deals with the transformation of the isochrones to many photometric systems, which require updating and extending the libraries of stellar spectra previously in use. The inclusion of dust reprocessing in the predicted photometry is illustrated in Sect. 4. A preliminary comparison with observational data is done in Sect. 5. Section 6 describes how to access the data tables - containing isochrones, bolometric

\footnotetext{
2 Marigo et al. (2003a) also computed isochrones containing essentially the same kind of TP-AGB tracks as in Cioni et al. (2006), but for a more limited range of metallicities, and for exploratory choices of model parameters. These isochrones were provided a few times upon request, and were superseded first by Cioni et al.'s and now by our new isochrones.
} 
corrections, dust attenuation curves, integrated magnitudes of single-burst populations, etc. - and summarizes both the main novelties and caveats of our new isochrones.

\section{From evolutionary tracks to isochrones}

\subsection{Putting tracks together}

The evolutionary tracks used in this work have already been thoroughly presented in previous papers. The evolution from the ZAMS up to the first thermal pulse on the AGB is taken from Gi00 for the initial metallicities $Z=0.0004,0.001,0.004,0.008$, 0.019 (the solar value), 0.03, and from Girardi (2001, unpublished; see http: //pleiadi . oapd. inaf.it) for $Z=0.0001$. The range of initial masses of these tracks goes from $M_{\mathrm{i}}=0.15$ to $7 M_{\odot}$, out of which all models between $M_{\mathrm{i}} \sim 0.5$ and $5 M_{\odot}$ are considered to undergo the TP-AGB phase.

The TP-AGB phase is then computed via a synthetic code as described in Paper I, starting from the physical conditions at the first thermal pulse, and up to the complete loss of the envelope via stellar winds. The model takes into account many critical aspects of the TP-AGB evolution, suh as the third dredge-up events; HBB over-luminosity and nucleosynthesis; pulse cycle luminosity and $T_{\text {eff }}$ variations; opacity variations driven by the changing chemical composition in the envelope; long-period variability (LPV) in both first overtone and fundamental modes; and their modulation of the mass-loss rates $\dot{M}$ via changes in the pulsation period $P$.

Especially relevant for this paper are the formalisms adopted for the mass loss: besides affecting the AGB lifetimes, high mass-loss rates determine the formation of circumstellar dust and hence the obscuration of AGB stars in the optical and their brightening in the mid- and far-IR (see Sect. 4). As detailed in Paper I, for O-rich AGB stars we use the results of dynamical atmospheres including dust for long-period variables calculated by Bowen (1988) and Bowen \& Willson (1991), taking into account whether pulsation occurs on the first overtone or on the fundamental mode. For C-rich stars, we assume two different regimes of mass loss: initially stars follow the semi-empirical relation from Schröder et al. (2003), then switching, above a critical luminosity, to the $\dot{M}$ values derived from the pulsating dust-driven wind models by Winters et al. (2000, 2003). In both cases, mass-loss rates depend on the pulsation period, which is predicted along the evolutionary tracks according to the periodmass-radius relations derived by Ostlie \& Cox (1986), Fox \& Wood (1982), Wood et al. (1983).

We replace the most massive TP-AGB tracks $\left(M_{\mathrm{i}} \geq 3.0 M_{\odot}\right)$ of Paper I by new calculations, accounting for a better description of the transition luminosity between the first-overtone and the fundamental-mode pulsation. This aspect is thoroughly described in the Appendix.

The synthetic TP-AGB evolution of Paper I has been followed up to the complete ejection of the stellar envelopes, hence including a good fraction of the post-AGB phase prior to the planetary nebula regime, i.e. the fast terminal stages during which the star evolves at almost constant luminosity, while $T_{\text {eff }}$ rapidly increases due to the combined effect of the outward displacement of the H-burning shell, and stripping of the residual envelope by stellar winds. These final parts of the tracks may not be reliable since some relations used in the synthetic TP-AGB code become inaccurate. Moreover, the mass-loss rates are critical in determining the duration of this post-AGB transition phase, but the rates are still poorly known.
Anyway, this transition phase turns out to be very short-lived $\left(\lesssim 10^{4} \mathrm{yr}\right.$ for low-mass stars, and even shorter for intermediatemass ones) if compared to the previous TP-AGB evolution. Therefore, although we mostly include this post-AGB phase in the new isochrones, its overall impact on them is almost negligible.

Another important detail is that before building the isochrones, TP-AGB tracks are "cleaned" of their luminosity and $T_{\text {eff }}$ variations caused by thermal pulse cycles. We only keep the stages of quiescent $\mathrm{H}$-shell burning previous to He-shell flashes, along with the stage of coolest $T_{\text {eff }}$, are kept for the isochrone interpolation. This is a necessary approximation since the large luminosity and $T_{\text {eff }}$ variations between confining tracks would lead to wild variations in the interpolated isochrones. Anyway, we can easily introduce the detailed evolution of luminosity and $T_{\text {eff }}$ over the thermal pulse cycles in the tabulated isochrones a posteriori, since the shape of thermal pulse cycles is a known function of other quantities that are also interpolated and stored along the isochrones, like the core mass and surface chemical composition (see, e.g., Wagenhuber \& Groenewegen 1998).

The Gi00 and Paper I sets of evolutionary tracks differ in their source of low-temperature $\left(T \lesssim 10^{4} \mathrm{~K}\right)$ opacities: while Gi00 uses tables for scaled-solar chemical compositions taken from Alexander \& Ferguson (1994), in Paper I the opacities are computed "on-the-fly" with the proper chemical mixture of the evolving TP-AGB envelopes, by means of the methods and approximations described in Marigo (2002). There is no dramatic change in opacities, however, in passing from the scaled-solar tables of Alexander \& Ferguson (1994) to Marigo's (2002) formalism, provided that the passage occurs at $\mathrm{C} / \mathrm{O}$ ratios much smaller than 1, as indeed is the case at the first thermal pulse of all TP-AGB tracks. The molecular opacities depart from those in Alexander \& Ferguson (1994) only later in the TP-AGB evolution, when the third dredge-up sets in and the $\mathrm{C} / \mathrm{O}$ ratio increases above 1 . Therefore, the concatenation of both sets of tracks at the stage of first thermal pulse produces only modest discontinuities in the tracks, i.e. small jumps in $T_{\text {eff }}$ that are caused by the small differences in opacities for O-rich mixtures ${ }^{3}$. For other quantities, like the luminosity, core mass, and surface chemical composition, the inspection of isochrones does not reveal any discontinuity.

Although the reader may feel uncomfortable with the small discontinuities in $T_{\text {eff }}$ at the stage of first thermal pulse in the isochrones, we remark that they are dramatically less important than any discontinuity that one may find when amalgamating tracks from completely different sources. The simple fact that the core mass and envelope chemical composition vary in a smooth and continuous way, for instance, ensures that the total emitted light derived from a given set of isochrones will be fully consistent with the chemical yields and total remnant masses derived from the same sets of tracks. As illustrated by Marigo \& Girardi (2001), this is an important, although largely ignored, consistency requirement to evolutionary population synthesis models of galaxies.

\subsection{Resulting isochrones}

Theoretical isochrones are built via simple linear interpolations inside the grids of evolutionary tracks, performed between "equivalent evolutionary points". The final isochrone, for a given age $t$, is made of a sample of such points conveniently distributed

\footnotetext{
3 These jumps in $T_{\text {eff }}$ can be appreciated in Fig. 1, at the points where the TP-AGB phase begins (i.e., in passing from green to blue lines).
} 
on the HR diagram. For each point, several stellar parameters are stored. In fact, the newest TP-AGB tracks take into account various aspects of evolution that have been ignored while making the GiO0 isochrones, but that are essential components of the TP-AGB models of Paper I, namely: surface chemical composition (especially the $\mathrm{C} / \mathrm{O}$ ratio), stellar core and envelope mass, mass-loss rates, pulsation modes and periods. These parameters can, for instance, allow easy identification of C-type and dustobscured stars, and are essential to the simulation of variations in stellar properties due to thermal pulse cycles and long-period variability. Therefore, our isochrone-making routines have been extended to take explicit care of these properties.

When an isochrone is built out of our tabulated tracks, mass loss and LPV pulsation are taken into detailed account only after the transition from the early-AGB to the TP-AGB regime. Variables, such as mass-loss rates, pulsation periods, and circumstellar dust emission, pass from "non-defined" to welldefined values, corresponding to the occurrence of the first thermal pulse. In order to avoid discontinuities in these variables, we have a posteriori evaluated the mass-loss rates and pulsation properties of stars in earlier phases, even if these have been computed at constant mass. The procedure is straightforward: for each star in the pre-TP-AGB section of the isochrones, the basic stellar properties $\left(L, T_{\mathrm{eff}}, M_{\mathrm{i}}\right)$ are used to determine pulsation periods and mass-loss rates in the same way as for O-rich AGB stars. Notice that in most cases mass loss prior to the TP-AGB phase is expected to be very modest and not have evolutionary implications, so that this procedure is well justified. The only exception is for low-mass models close to the tip of the red giant branch (TRGB), where the use of Reimers (1975) mass-loss formula with a multiplicative factor of $\eta=0.4$, leads to the loss of $\Delta M^{\mathrm{RGB}} \lesssim 0.2 M_{\odot}$ for low-mass stars ${ }^{4}$. This amount of mass lost is necessary to explain the HB morphologies observed in galactic globular clusters (see Renzini \& Fusi Pecci 1988). The use of the Reimers mass-loss formula for the RGB is seen as a "conservative choice" since, although alternative formulas exist (see Catelan 2000, 2007), currently none can be clearly favoured over the others. We take the RGB mass loss into consideration during the construction of stellar isochrones by simply jumping from a TRGB track of given mass $M$ to a ZAHB track of mass $M-\Delta M^{\mathrm{RGB}}$. The procedure is somewhat detailed in Bertelli et al. (1994) and in Gi00.

Extended sets of isochrones are depicted in the HR diagrams of Fig. 1. Since the main novelty of the present paper is in the detailed description of the TP-AGB phase, the HR diagrams are zoomed on this phase. In other parts of the HR, isochrones are simply identical to those previously computed by Gi00.

It is evident from the figure that our new isochrones reflect all of the peculiar behaviours of the TP-AGB tracks they derive from (see Fig. 20 in Paper I). In particular, we notice the following:

At moderate-to-high metallicities, i.e. for $Z \geq 0.004$ : in the youngest isochrones, the combination of HBB and high massloss rates gives origin to bell-shaped TP-AGB curves. The luminosity first rapidly grows above the core-mass luminosity relation because of the occurrence of HBB, then higher mass-loss rates are attained, drastically reducing the stellar envelope mass so that $\mathrm{HBB}$ and the related over-luminosity are eventually extinguished.

${ }^{4}$ The behaviour of $\Delta M^{\mathrm{RGB}}$ as a function of age can be appreciated in Fig. 6 of Girardi (1999), top panel.
These stars usually have $\mathrm{C} / \mathrm{O}<1$, although a late transition to the $\mathrm{C}$-rich phase may take place due to final dredge-up events when HBB has already ceased (see Frost et al. 1998) in agreement with observations (e.g. van Loon et al. 1998, 1999).

The C-star phase is completely developed in isochrones of intermediate ages, and it happens at very low $T_{\text {eff }}$ (reaching $T_{\text {eff }} \simeq 2500 \mathrm{~K}$ ), in a sort of "red tail" which is neatly separated from the sequences of O-rich stars. This separation naturally results from the very different low-temperature opacities of C-rich envelopes (Marigo 2002).

In the oldest isochrones, dredge-up is not operating and the TP-AGB sections of the isochrones simply align above the previous early-AGB phase. They steadily increase in luminosity and slightly bend to the left at their upper end, as a result of the progressive thinning of stellar envelopes.

At low metallicities, i.e., for $Z<0.004$ : some of the previous trends change. One important difference is that, despite the high efficiency of $\mathrm{HBB}$, a C-star phase is present even at younger ages. This is caused by the activation of the ON cycle which converts $\mathrm{O}$ into $\mathrm{N}$, so that the $\mathrm{C} / \mathrm{O}$ ratio is predicted to increase above unity (Ventura et al. 2002; and Paper I). Compared to the tracks of higher metallicities, the C-star sequences start at higher effective temperatures, as expected from the lower molecular concentrations in their atmospheres. The oldest O-rich sequences evolve to larger luminosities while bending to the left at their high ends, just as in the tracks for higher metallicities. This feature is essentially related to the outward displacement of the $\mathrm{H}$-burning shell, which is found to be more efficient than the stellar winds in reducing the envelope mass of low-mass AGB stars $\left(M_{\mathrm{i}} \lesssim 1 . M_{\odot}\right)$.

A more detailed discussion of the above-mentioned behaviors is presented in Paper I.

In order to better illustrate the peculiarities of the TP-AGB models presently available, Fig. 2 presents a series of $Z=0.008$ isochrones derived from Gi00 tracks connected with different extensions of the TP-AGB phase. In the first isochrones (Gi00), the TP-AGB phase is simplified and merely extends the previous evolution straight above the RGB and early-AGB phases, without any $\mathrm{C}$-star sequence and without any signature of HBB. Then, the Marigo \& Girardi (2001) isochrones introduce dredge-up and $\mathrm{HBB}$ in the TP-AGB, and present a well-defined $\mathrm{C}$-star region, which is still aligned on top of the RGB and early-AGB, as expected from models with opacities for scaled-solar compositions. Cioni et al. (2006) introduce variable molecular opacities and then the C-star sequence clearly changes its shape on the HR diagram (as explained by Marigo 2002). Finally, the sequence of Fig. 2 could be completed with the third panel of Fig. 1, which represents the present state of the $Z=0.008$ isochrones, differing from the Cioni et al. (2006) in that the C-star luminosities and lifetimes are now calibrated (see Paper I).

\section{Transformations to several photometric systems}

The new isochrones are transformed to several photometric systems, following the formalism described in Girardi et al. (2002) to derive bolometric corrections from a library of stellar spectra. Although this argument will be more thoroughly discussed in a forthcoming paper (Girardi et al. 2007, in preparation), it is worth recalling the main steps we follow to deal with the novelties of the present release. 

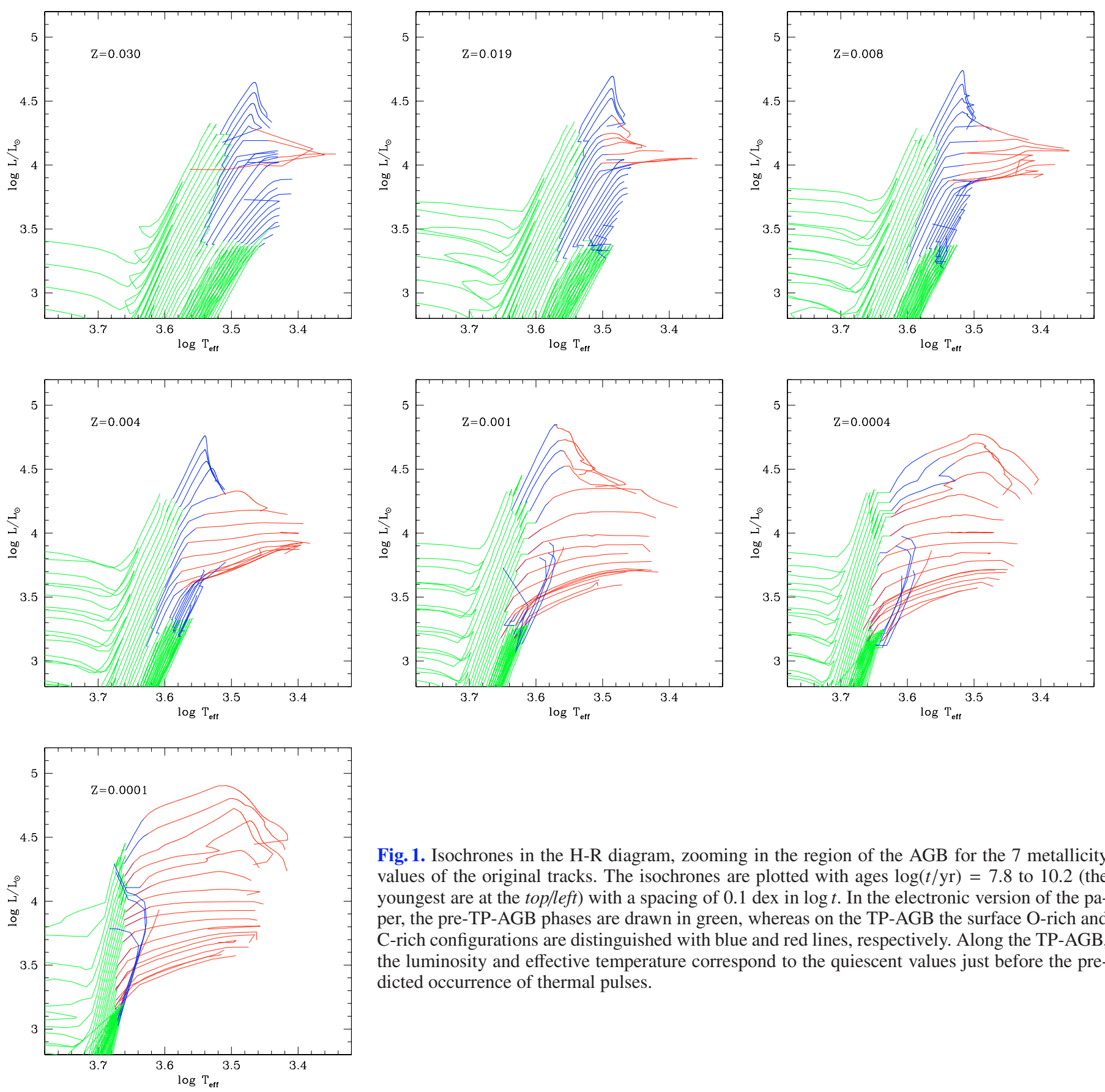

Fig. 1. Isochrones in the H-R diagram, zooming in the region of the AGB for the 7 metallicity values of the original tracks. The isochrones are plotted with ages $\log (t / \mathrm{yr})=7.8$ to 10.2 (the youngest are at the top/left) with a spacing of 0.1 dex in $\log t$. In the electronic version of the paper, the pre-TP-AGB phases are drawn in green, whereas on the TP-AGB the surface O-rich and $\mathrm{C}$-rich configurations are distinguished with blue and red lines, respectively. Along the TP-AGB, the luminosity and effective temperature correspond to the quiescent values just before the predicted occurrence of thermal pulses.

\subsection{Bolometric corrections and extinction coefficients}

Given the spectral flux at the stellar surface, $F_{\lambda}$, bolometric corrections for any set of filter transmission curves $S_{\lambda}$ are given by (see Girardi et al. 2002, for details):

$$
\begin{aligned}
B C_{\mathrm{S}_{\lambda}}= & M_{\mathrm{bol}, \odot}-2.5 \log \left[4 \pi(10 \mathrm{pc})^{2} F_{\mathrm{bol}} / L_{\odot}\right] \\
& +2.5 \log \left(\frac{\int_{\lambda_{1}}^{\lambda_{2}} \lambda F_{\lambda} 10^{-0.4 A_{\lambda}} S_{\lambda} \mathrm{d} \lambda}{\int_{\lambda_{1}}^{\lambda_{2}} \lambda f_{\lambda}^{0} S_{\lambda} \mathrm{d} \lambda}\right)-m_{\mathrm{S}_{\lambda}}^{0},
\end{aligned}
$$

where $f_{\lambda}^{0}$ represents a reference spectrum (at the Earth) that produces a known apparent magnitude $m_{\mathrm{S}_{\lambda}}^{0}$, and $F_{\text {bol }}=\int_{0}^{\infty} F_{\lambda} \mathrm{d} \lambda=$ $\sigma T_{\text {eff }}^{4}$ is the total emerging flux at the stellar surface. Once $B C_{\mathrm{S}_{\lambda}}$ are computed, stellar absolute magnitudes follow from

$M_{\mathrm{S}_{\lambda}}=M_{\mathrm{bol}}-B C_{\mathrm{S}_{\lambda}}$, where

$$
\begin{aligned}
M_{\mathrm{bol}} & =M_{\mathrm{bol}, \odot}-2.5 \log \left(L / L_{\odot}\right) \\
& =M_{\mathrm{bol}, \odot}-2.5 \log \left(4 \pi R^{2} F_{\mathrm{bol}} / L_{\odot}\right) .
\end{aligned}
$$

As in Girardi et al. (2002), we adopt $M_{\text {bol, } \odot}=4.77$, and $L_{\odot}=$ $3.844 \times 10^{33} \mathrm{erg} \mathrm{s}^{-1}$ (Bahcall et al. 1995).

Notice that Eq. (1) uses photon count integration instead of energy integration, as adequate to most modern photometric systems which use photon-counting devices instead of energy-amplifiers.

The equation also includes the interstellar extinction curve $A_{\lambda}$, which we set to $A_{\lambda}=0$. For the optical and near-IR, we provide the extinction coefficients $A_{\lambda} / A_{\mathrm{V}}$ as computed for a yellow dwarf of solar metallicity (the Sun) using the Cardelli et al. (1989) $R_{\mathrm{V}}=3.1$ extinction curve. The small variations of 

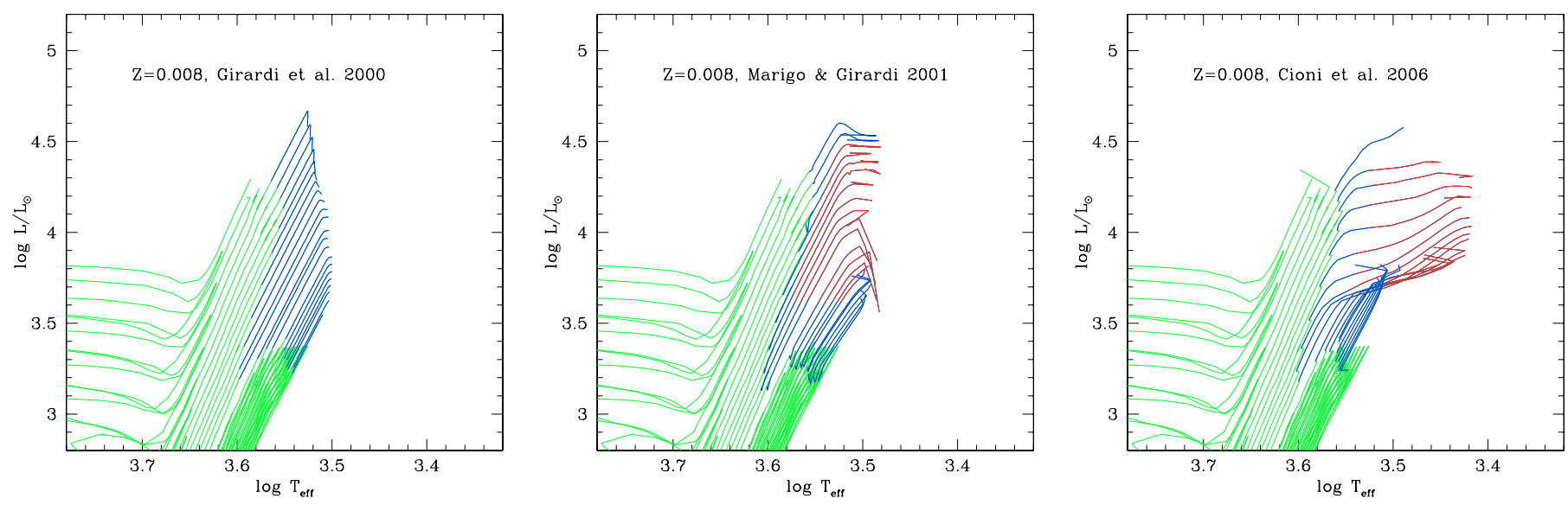

Fig. 2. The same as in Fig. 1, but for a sequence that illustrates the evolution of the TP-AGB phase since the release of Gi00 isochrones. From left to right, isochrones are from Gi00, Marigo \& Girardi (2001), and Cioni et al. (2006). This sequence would be completed with the third panel of Fig. 1.

such coefficients with spectral type and extinction (see Grebel \& Roberts 1995) will be discussed in a separate paper (Girardi et al., in prep.) that will detail the transformations to WFPC2 and ACS systems, and describe a way to include consistently the interstellar extinction into the isochrones.

\subsection{Assembling a library of spectral fluxes}

Girardi et al. (2002) describes the assembly of a large library of spectral fluxes, covering wide ranges of initial metallicities ([M/H], from -2.5 to +0.5 ), $T_{\text {eff }}$ (from 600 to $50000 \mathrm{~K}$ ), and $\log g$ (from -2 to 5$)^{5}$. This grid is wide enough to include most of the spectral types that constitute the bulk of observed samples. It is composed of ATLAS9 "NOVER" spectra from Castelli et al. (1997; see also Bessell et al. 1998) for most of the stellar types; Allard et al. (2000; see also Chabrier et al. 2000) for M, $\mathrm{L}$, and T dwarfs ${ }^{6}$; Fluks et al. (1994) empirical spectra for M giants; and, finally, pure black-body spectra for stars exceeding $50000 \mathrm{~K}$.

Since then this library been revised. We have replaced the ATLAS9 spectra by the newest "ODFNEW" ones from Castelli \& Kurucz (2003), which incorporate several corrections to the atomic and molecular data, especially regarding the molecular line lists of $\mathrm{CN}, \mathrm{OH}, \mathrm{SiO}, \mathrm{H}_{2} \mathrm{O}$ and $\mathrm{TiO}$, and the inclusion of $\mathrm{HI}-\mathrm{H}+$ and $\mathrm{HI}-\mathrm{H}+$ quasi-molecular absorption in the UV. These new models are considerably better than previous ones in the $\mathrm{UV}$ region of the spectrum, and over most of the wavelength range for $T_{\text {eff }}$ between $\sim 4500$ and $3500 \mathrm{~K}$ (see Figs. 1 to 3 in Castelli \& Kurucz 2003).

Moreover, the library has been extended with the Loidl et al. (2001) synthetic C-star spectra for $3600 \gtrsim\left(T_{\text {eff }} / \mathrm{K}\right) \gtrsim 2600$, derived from hydrostatic model atmospheres computed with MARCS (see also Höfner et al. 2003, for more details). For C-stars cooler than $2600 \mathrm{~K}$, we simply adopt the BC values derived from $2600 \mathrm{~K}$ spectra. These Loidl et al. (2001) spectra are computed for $\mathrm{C} / \mathrm{O}=1.1$ for all $T_{\text {eff }}$, and for both $\mathrm{C} / \mathrm{O}=1.1$ and 1.4 for $T_{\text {eff }} \leq 3200 \mathrm{~K}$. We have verified that bolometric

\footnotetext{
${ }^{5}$ In this paper, the surface gravity $g$ is expressed in cgs units.

6 Although brown dwarfs are not the subject of this paper, the tables of bolometric corrections include these objects. More details can be found in the original paper by Allard et al. (and to a smaller extent also Girardi et al. 2002).
}

corrections for all broadbands redder than $V$ are quite insensitive to the $\mathrm{C} / \mathrm{O}$ ratio, varying by less than 0.1 mag between the $\mathrm{C} / \mathrm{O}=1.1$ and 1.4 cases. These differences become $\lesssim 0.01 \mathrm{mag}$ in the $J$-band. For the bluest optical pass-bands the choice between these two $\mathrm{C} / \mathrm{O}$ values causes significant differences in the bolometric corrections, amounting for instance to $\gtrsim 0.4$ mag in $B$. The $T_{\text {eff }} \leq 3200 \mathrm{~K} \mathrm{C}$-stars are much fainter (more than 4 mag in a Vega system) at these blue wavelengths than at the $I$-band, and therefore these cases are of little practical interest. Hence, we adopt by default the $\mathrm{C} / \mathrm{O}=1.1 \mathrm{C}$-star spectra, for all $T_{\text {eff }}$. We will follow a more elaborate approach when spectra for a wider range of $\mathrm{C} / \mathrm{O}$ values become available (Aringer et al., in preparation).

Finally, as part of the TRILEGAL project (Girardi et al. 2005), we have included spectra for DA white dwarfs from Finley et al. (1997) and Homeier et al. (1998) for $T_{\text {eff }}$ between 100000 and $5000 \mathrm{~K}$, and $\log g$ between 7 and 9.

The Castelli \& Kurucz (2003) and Allard et al. (2000) tabulated spectra extend well into the far-IR, until 160 and $971 \mu \mathrm{m}$ respectively. In the other cases, the available spectra were only tabulated to the mid-IR, namely until $12.5 \mu \mathrm{m}$ for Fluks et al. (1994) and $2.5 \mu \mathrm{m}$ for Loidl et al. (2001). In all cases, we have extended the flux to the very far-IR with a scaled Rayleigh-Jeans law starting from the point of maximum tabulated $\lambda$. This is clearly a crude approximation. For instance, van der Bliek et al. (1996) find that blackbody fluxes in the far-IR present discrepancies of the order of 20 per cent with respect to the solar flux. Decin \& Eriksson (2007) recently discussed in detail the status of the predicted spectral energy distributions in the IR. In light of their results, our predicted BC for the mid- and far-IR will be uncertain by up to a few tenths of a magnitude for the coolest $\mathrm{M}$ and $\mathrm{C}$ stars. However, we expect that the errors in colours will be somewhat smaller, and they will become irrelevant in stars with significant mass-loss, which are exactly those with the most prominent fluxes in the mid- and far-IR. In fact, in these cases, the emission at mid- and far-IR wavelengths is dominated by the circumstellar dust shells and not by the stellar photosphere (see Sect. 4).

\subsection{Filter sets so far considered}

Table 2 presents all the photometric systems we have considered so far. They include several traditional ground-based 
photometric systems (Johnson-Cousins-Glass, StrömgrenCrawford, Washington, BATC), systems belonging to some influential wide-field surveys (DENIS, 2MASS, OGLE-II, SDSS) and cameras (WFI, UKIDSS), and then to some of the most successful satellite-based cameras of the recent past (Tycho, ACS) or still in operation (WFPC2, NICMOS, Spitzer, AKARI). Other systems are presently being added to the list.

Note that the transformations to some of these photometric systems are described in other papers (e.g., Girardi et al. 2007, for WFPC2 and ACS; Vanhollebeke et al. 2007, for OGLE-II); they are listed in Table 2, and no further detail on these systems will be provided here. All systems in the Table are already available at the web interface (see Sect. 6.1).

\section{Reprocessing by circumstellar dust}

Circumstellar dust reprocesses a substantial fraction of the photons emitted by the coolest evolved stars. Since, on one hand, the efficiency of dust extinction decreases quickly above $\sim 1 \mu \mathrm{m}$ and, on the other hand, dust does not survive at temperatures above $1000-2000 \mathrm{~K}$, the general effect of the presence of dust in an astrophysical source is to shift part of the intrinsic emitted power from the optical-ultraviolet to the mid- and far-infrared region.

A serious difficulty in taking into account in detail the effects of dust is that the optical properties (i.e., the efficiency of absorption and scattering as a function of wavelength) differ significantly in different environments. This is particularly true for the envelopes of AGB stars, which are believed to be one of the two main producers of interstellar grains, the other being the supernovae blast waves (e.g., Dwek 1998; Todini \& Ferrara 2001; Morgan \& Edmunds 2003; Calura et al. 2007). As for the dust composition, the general wisdom is that the envelopes of C-type stars are composed of carbonaceous grains while those of M-type stars are dominated by silicates, however several recent detailed studies, mainly based on Spitzer data (e.g., Lebzelter et al. 2006; Verhoelst et al. 2006) and in more detailed modelling (e.g., Ireland \& Scholz 2006; Woitke 2006; Ferrarotti \& Gail 2006) demonstrated that the situation may be more complex. Moreover, the grain size distribution, which together with the composition determines the optical properties, is expected to be significantly different from that inferred in the general interstellar medium.

A further big difficulty in the understanding of AGB envelopes is the occurrence of strong mass loss and the possible relation with the type of dust that can condensate in their outflows. The massive winds in AGB stars are commonly ascribed to the coupled effect between large amplitude pulsations and radiation pressure on dust grains. According to detailed dynamical studies, in C-rich stars the radiation pressure on dust can in fact be strong enough to explain the observed outflows (see e.g., Winters et al. 2000; Höfner et al. 2003). Instead in O-rich stars the possible type of grains are either not stable or opaque enough near $\sim 1 \mu \mathrm{m}$ to explain the outflows (e.g., Ireland \& Scholz 2006; Woitke 2006).

Furthermore, recent spectroscopic analyses of oxygen-rich AGB stars based on ISO data for the LMC (Dijkstra et al. 2005) and the Galactic Bulge (Blommaert et al. 2006) have shown a clear correlation between the dust composition and the massloss rates. For instance, the dust features displayed by oxygenrich stars are mainly ascribed to $\mathrm{Al}_{2} \mathrm{O}_{3}$ for low $\dot{M}$, while going to higher $\dot{M}$ they are interpreted with an increasing amount of silicates. These findings are consistent with the latest results from Spitzer data of AGB stars belonging to the globular cluster 47 Tuc (Lebzelter et al. 2006). The dust mineralogy actually changes as the star evolves at increasing luminosities and pulsation periods. On the other hand, for $\mathrm{C}$ stars unveiling the dust-condensation sequence is more problematic due to its complex dependence on a number of factors (Speck et al. 2006; Thompson et al. 2006).

What emerges is that, despite conspicuous progress in both observational and theoretical grounds, the issue of dust formation and evolution in AGB stars is still affected by large uncertainties. For these reasons in this paper we will explore a few kinds of circumstellar dust, essentially following the prescriptions by Groenewegen (2006, G06) and Bressan et al. (1998, B98). Alternative and more complex descriptions will be examined in subsequent papers.

\subsection{Method}

This section outlines the adopted procedure to include the effect of dust in our isochrones. In general, for a given star of ( $\left.L, T_{\text {eff }}\right)$, losing mass at a rate $\dot{M}$, with dust expansion velocity $v_{\exp }$ and dust-to-gas ratio $\Psi$, radiation transfer (RT) calculations provide the dust optical depth $\tau$ and its corresponding attenuation/emission spectrum. The optical depth $\tau$ scales with the other quantities via the approximated relation

$\tau(1 \mu \mathrm{m}) \simeq A_{\mathrm{d}} \dot{M} \Psi v_{\text {exp }}^{-1} L^{-0.5}$,

(see G06 and references therein) where $A_{\mathrm{d}}$ is a quantity that is set mainly by the dust composition.

For convenience, we have pre-computed a number of reference $\mathrm{R}$ models parametrized as a function of a basic set of parameters, namely: $\tau(1 \mu \mathrm{m}), T_{\text {eff }}, L$, dust condensation temperature $T_{\mathrm{c}}$, and for different dust mixtures, as specified in Sects. 4.4.1 and 4.4.2 for G06 and B98 models, respectively. Then, each star along the isochrone is assigned its proper dusty envelope by using Eq. (4), with the corresponding values of $\dot{M}$, $\Psi, v_{\text {exp }}, L, T_{\text {eff }}$, and $A_{\mathrm{d}}$.

Each determination of $\tau(1 \mu \mathrm{m})$ corresponds to specific dust attenuation and emission functions, which in turn fix the ratio between the flux derived from RT calculations and the dust-free intrinsic flux, i.e., $F_{\lambda} / F_{\lambda, 0}$. Finally, bolometric corrections are derived, i.e. $\Delta \mathrm{BC}_{\lambda}=-2.5 \log \left(F_{\lambda} / F_{\lambda, 0}\right)$.

In summary, for each AGB star along the isochrone, the general scheme proceeds through the following steps:

- we first distinguish between O- and C-rich envelopes, using the photospheric $\mathrm{C} / \mathrm{O}$ ratio. The dust composition is selected among those for which RT calculations have been performed;

- $v_{\text {exp }}$ and $\Psi$ are computed as a function of $L, T_{\text {eff }}, \mathrm{C} / \mathrm{O}$ and $\dot{M}$, using the formalism described in Sects. 4.2 and 4.3 below;

- the proper $\tau$ is calculated via Eq. (4);

- the corresponding $\Delta \mathrm{BC}_{\lambda}$ relation is interpolated at the effective wavelengths $\lambda_{\text {eff }}$ of all filters considered in our isochrones. The resulting $\Delta \mathrm{BC}_{\lambda_{\text {eff }}}$ values are added to the $\mathrm{ab}-$ solute magnitudes computed previously via Eq. (1).

\subsection{Expansion velocity}

The wind terminal velocity or expansion velocity, $v_{\text {exp }}$, is calculated according to the formalism presented by Elitzur \& Ivezić (2001), to which the reader should refer for the details of the underlying theoretical analysis. In brief, following Elitzur \& Ivezić (2001) the dust-wind problem is solved by means of a suitable scaling approach, so as it is possible to express $v_{\exp }$ as a function of mass-loss rate, stellar luminosity, and various dust parameters 


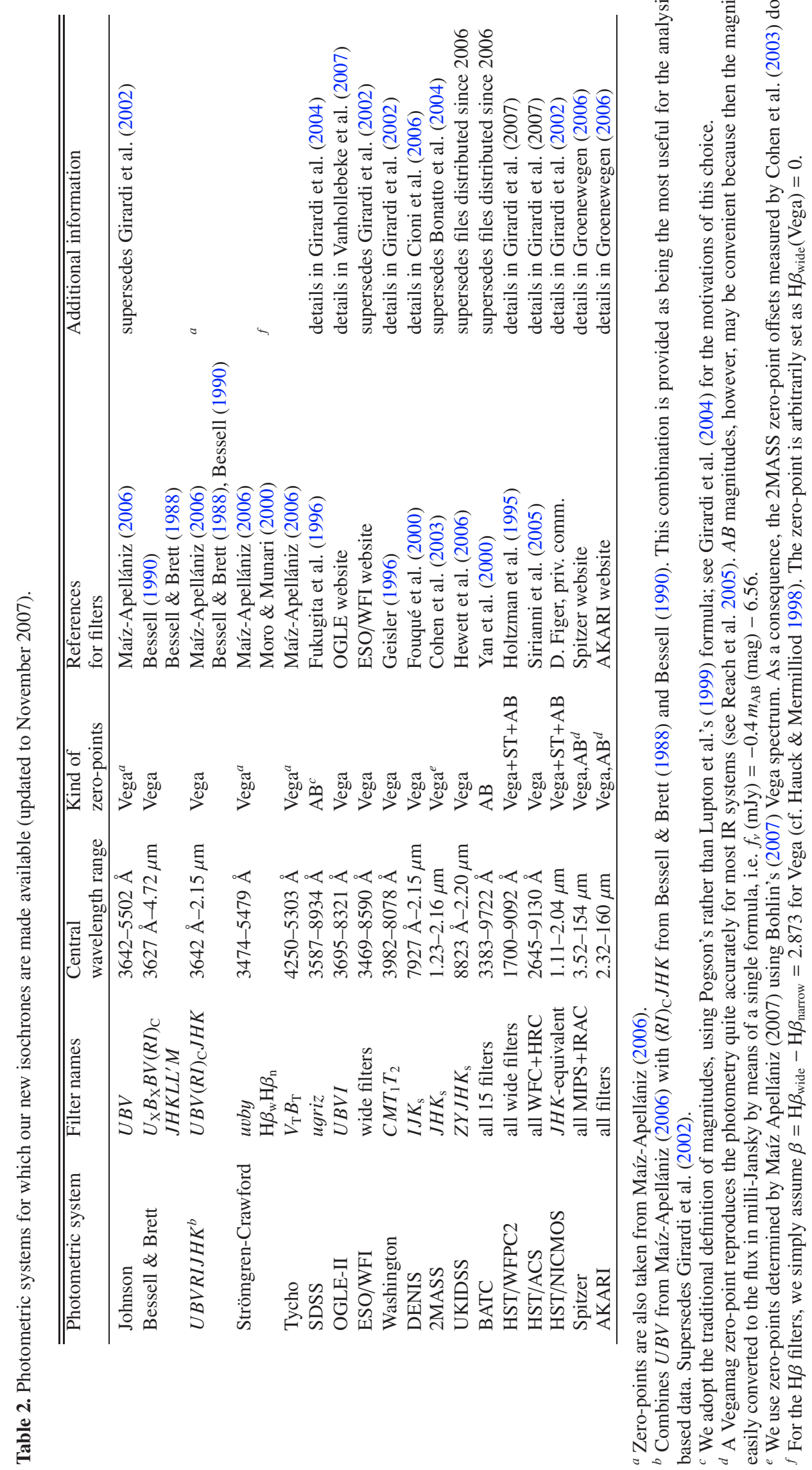


(dust-to-gas ratio, chemical composition, condensation temperature, etc.) in the form,

$v_{\exp }=\left(A \dot{M}_{-6}\right)^{1 / 3}\left(1+B \frac{\dot{M}_{-6}^{4 / 3}}{L_{4}}\right)^{-1 / 2}$,

where $v_{\exp }$ is expressed in $\mathrm{km} \mathrm{s}^{-1} ; \dot{M}_{-6}$ is the mass-loss rate in units of $10^{-6} M_{\odot} \mathrm{yr}^{-1} ; L_{4}$ is the stellar luminosity in units of $10^{4} L_{\odot}$.

The dependence of $v_{\text {exp }}$ on the dust-to-gas ratio is included in the definition of the parameters $A$ and $B$ (through the quantity $\sigma_{22}$, see below):

$A=3.08 \times 10^{5} T_{\mathrm{c} 3}^{4} Q_{\star} \sigma_{22}^{2} \chi_{0}^{-1}$,

$B=\left[2.28 \frac{Q_{\star}^{1 / 2} \chi_{0}^{1 / 4}}{Q_{V}^{3 / 4} \sigma_{22}^{1 / 2} T_{\mathrm{c} 3}}\right]^{-4 / 3}$

which in turn depend on several quantities quoted here below. The condensation temperature in units of $10^{3} \mathrm{~K}$ is denoted with $T_{\mathrm{c} 3}$. The parameter $Q_{\star}$ is the Planck average of the efficiency coefficient for radiation pressure, evaluated at the effective temperature, while $Q_{V}$ is the efficiency factor for absorption at visual. The quantity $\chi_{0}$ is the ratio:

$\chi_{0}=\frac{Q_{\mathrm{P}}\left(T_{\mathrm{eff}}\right)}{Q_{\mathrm{P}}\left(T_{\mathrm{c}}\right)}$,

where $Q_{\mathrm{P}}(T)$ is the Planck average of the absorption efficiency at the temperature $T$.

We define $\sigma_{22}=\sigma_{\text {gas }} /\left(10^{-22} \mathrm{~cm}^{2}\right)$, where $\sigma_{\text {gas }}\left(\right.$ in $\left.\mathrm{cm}^{2}\right)$ is the dust cross-sectional area per gas particle at condensation

$\sigma_{\text {gas }}=\pi a^{2} \frac{n_{\text {dust }}}{n_{\text {gas }}}$.

In the above equation $a$ is the mean size (in $\mathrm{cm}$ ) of the dust grains, $n_{\text {dust }}$ and $n_{\text {gas }}$ are the number densities (in $\mathrm{cm}^{-3}$ ) of dust and gas particles, respectively. As in Elitzur \& Ivezić (2001), here it is assumed that all dust grains have the same size.

The dust-to-gas mass ratio is defined as

$\Psi=\frac{\rho_{\text {dust }}}{\rho_{\text {gas }}}$

where $\rho_{\text {dust }}$ and $\rho_{\text {gas }}$ correspond to the density (in $\mathrm{g} \mathrm{cm}^{-3}$ ) of the matter in the form of dust and gas, respectively.

Assuming that the dust grains have a specific density $\rho_{\text {grain }}$ (in $\mathrm{g} \mathrm{cm}^{-3}$ ), the explicit form of the dust density reads

$\rho_{\text {dust }}=\frac{4}{3} \pi a^{3} \rho_{\text {grain }} n_{\text {dust }}$.

With the approximation that all the gas is made up of hydrogen and helium, with abundances (in mass fraction) $X_{\mathrm{H}}$ and $X_{\mathrm{He}}$ respectively, then

$\rho_{\text {gas }}=A_{\text {gas }} m_{\mathrm{H}} n_{\text {gas }}$

where $A_{\text {gas }} \simeq 4 /\left(4 X_{\mathrm{H}}+X_{\mathrm{He}}\right)$ is the mean molecular weight of the gas in units of the mass of the $\mathrm{H}$ atom, $m_{\mathrm{H}}=1.674 \times 10^{-24} \mathrm{~g}$, and $n_{\text {gas }}$ is the number density of the gas particles $\left(\right.$ in $\mathrm{cm}^{-3}$ ).

Finally, inserting Eqs. (11) and (12) into Eq. (10), we can re-write Eq. (9) to show the dependence on the dust-to-gas ratio

$\sigma_{\text {gas }}=\frac{3}{4} \frac{A_{\text {gas }} m_{\mathrm{H}}}{a \rho_{\text {grain }}} \Psi$.
It is worth noting that in the Elitzur \& Ivezić (2001) formalism the dust-to-gas mass ratio is indeed a free parameter. In Sect. 4.3, we detail our scheme to specify $\Psi$ as a function of basic stellar parameters like $\dot{M}$ and the $\mathrm{C} / \mathrm{O}$ ratio.

Moreover, given the dust chemical type (either amorphous carbon or silicates) in Elitzur \& Ivezić (2001) the quantities $Q_{\mathrm{P}}$ and $Q_{\star}$ are assigned fixed values corresponding to a particular choice of the dust condensation temperature $\left(T_{\mathrm{c}}=800 \mathrm{~K}\right)$ and the stellar effective temperature $\left(T_{\text {eff }}=2500 \mathrm{~K}\right)$. We have carried out additional runs of DUSTY (Ivezić et al. 1999) to evaluate $Q_{\mathrm{P}}$ and $Q_{\star}$ for various values of $T_{\mathrm{c}}$ and $T_{\text {eff }}$, and for different dust compositions. The results are presented in Table 3 and are used in our calculations, by interpolating in $T_{\mathrm{eff}}$ and $T_{\mathrm{c}}$ and allowing for different dust mixtures. In the present paper, we adopt $T_{\mathrm{c}}=$ $1000 \mathrm{~K}$ when $\mathrm{C} / \mathrm{O}<1$ and $T_{\mathrm{c}}=1500 \mathrm{~K}$ when $\mathrm{C} / \mathrm{O}>1$. The grain size is set $a=0.1 \mu \mathrm{m}$ in all cases. The other input parameters, i.e., effective temperature and dust chemical type, are just those predicted by our stellar isochrones.

It should be also mentioned that Eq. (5) is valid as long as dust condensation is efficient enough to drive an outflow. As discussed in Elitzur \& Ivezić (2001) this condition corresponds to the minimum mass-loss rate:

$\dot{M}_{\min }=3 \times 10^{-9} \frac{M^{2}}{Q_{\star} \sigma_{22}^{2} L_{4} T_{k 3}^{1 / 2}} \quad\left(M_{\odot} \mathrm{yr}^{-1}\right)$

where $T_{\mathrm{k} 3}$ is the kinetic temperature in units of $10^{3} \mathrm{~K}$ at the inner boundary of the dust shell. For simplicity, we assume that the kinetic temperature $T_{\mathrm{k}}$ coincides with the condensation temperature $T_{\mathrm{c}}$ of the dust. Interestingly we have verified that $\dot{M}_{\text {min }}$ given by Eq. (14) is in good agreement with the results from hydrodynamical wind calculations, i.e., $\dot{M}_{\min } \approx 3-5 \times 10^{-7} M_{\odot} \mathrm{yr}^{-1}$ as predicted by Wachter et al. (2002) for C-stars.

For $\dot{M}<\dot{M}_{\text {min }}$, while dust is not a driving mechanism to produce a wind, it may be still formed passively in the circumstellar ejecta. To handle this case we have simply evaluated the expansion velocity setting $\dot{M}=\dot{M}_{\min }$ in the corresponding Eq. (5). In this way $v_{\exp }$ is seen to decline smoothly with the luminosity to the lowest measured values and, perhaps more importantly, by using $v_{\exp }=v_{\exp }\left(\dot{M}_{\min }\right)$ together with the actual $\dot{M}$ in Eq. (4), we can deal with the very optically-thin circumstellar envelopes $\left(\tau(1 \mu \mathrm{m}) \lesssim 10^{-2}-10^{-3}\right)$ expected at low $\dot{M}$.

One important feature of Eq. (5) is that it naturally predicts a maximum value for the expansion velocity, which is attained at large $\dot{M}$ as a consequence of the weakening of radiative coupling in optically-thick winds: $v_{\max } \simeq v_{\exp }\left(\tau_{\mathrm{V}}=1.3\right)$. This aspect may bring along significant implications for the interpretation of the observed data (Sect. 5.1; see Fig. 4).

\subsection{Dust-to-gas mass ratio}

Under the assumptions of steady-state, spherically symmetric, radial outflow with complete coupling between dust and gas (i.e. both components sharing the same velocity), from the massconservation law $\dot{M}=4 \pi r^{2} \rho v_{\text {exp }}$, the dust-to-gas mass ratio $\Psi$ - defined by Eq. (10) - can be expressed:

$\Psi=\frac{\dot{M}_{\text {dust }}}{\dot{M}_{\text {gas }}}$

where $\dot{M}_{\text {dust }}$ and $\dot{M}_{\text {gas }}=\dot{M}-\dot{M}_{\text {dust }}$ denote the mass-loss rates in the form of dust and gas, respectively. For any given combination of $\dot{M}$ and $\mathrm{C} / \mathrm{O}, \dot{M}_{\text {dust }}$ is calculated on the basis of the formalism presented by Ferrarotti (2003) and 
Table 3. Characteristic quantities used in the dust models, following the formalism developed by Elitzur \& Ivezić (2001). They are defined in Sect. 4.2.

\begin{tabular}{ccccccccc}
\hline \hline dust composition & $Q_{\mathrm{V}}$ & $\rho_{\text {grain }}\left(\mathrm{gr} \mathrm{cm}^{-3}\right)$ & $\mathrm{a}(\mu \mathrm{m})$ & $T_{\text {eff }}$ & $Q_{\star}\left(T_{\text {eff }}\right)$ & $Q_{P}\left(T_{\text {eff }}\right)$ & $T_{\mathrm{c}}$ & $Q_{P}\left(T_{\mathrm{c}}\right)$ \\
\hline 100\% AlOx & 0.370 & 3.2 & 0.1 & 2000 & 0.0189 & 0.0087 & 1500 & 0.00811 \\
& & & & 2500 & 0.0344 & 0.0105 & 1200 & 0.00897 \\
& & & & 3000 & 0.0599 & 0.0128 & 1000 & 0.0107 \\
& & & & 3500 & 0.0958 & 0.0157 & 800 & 0.0145 \\
\hline 100\% Silicate & 0.779 & 3.0 & 0.1 & 2000 & 0.0688 & 0.0531 & 1500 & 0.0396 \\
& & & & 2500 & 0.106 & 0.0696 & 1200 & 0.0333 \\
& & & & 3000 & 0.159 & 0.0884 & 1000 & 0.0303 \\
& & & 3500 & 0.231 & 0.110 & 800 & 0.0291 \\
\hline 100\% Silicon Carbide & 5.17 & 3.2 & 0.1 & 2000 & 0.148 & 0.0825 & 1500 & 0.0576 \\
& & & & 2500 & 0.293 & 0.120 & 1200 & 0.0438 \\
& & & & 3000 & 0.527 & 0.176 & 1000 & 0.0348 \\
& & & 3500 & 0.847 & 0.249 & 800 & 0.0264 \\
\hline
\end{tabular}

Ferrarotti \& Gail (2006, and references therein), to whom we refer for many details. The general scheme is the following:

$\dot{M}_{\text {dust }}=\sum_{\mathrm{i}} \dot{M}_{\text {dust }, i}$

where the summation includes the contribution of various dust species, each one being expressed

$\dot{M}_{\text {dust }, \mathrm{i}}=\dot{M} X_{\text {seed }}\left(\frac{A_{\text {dust }, i}}{A_{\text {seed }}}\right) f_{\text {dust }, i}$,

where $X_{\text {seed }}$ is the total mass fraction of the seed ${ }^{7}$ element in the circumstellar envelope, $A_{\text {seed }}$ denotes its atomic weight, whereas $A_{\text {dust,i }}$ corresponds to the mean molecular weight of the dust species. The adopted values are taken from Ferrarotti \& Gail (2002). The quantity $f_{\text {dust, } i}$ is the condensation degree of the dust species, being defined as the fraction of the seed element condensed into dust grains. In our calculations, the condensation degrees are estimated as a function of the total mass-loss rate and $\mathrm{C} / \mathrm{O}$ ratio by means of the analytic fits provided in Ferrarotti (2003).

The types of dust species that may condensate in the AGB circumstellar envelopes depend critically on the available abundances of the main dust forming elements, i.e., $\mathrm{C}, \mathrm{O}, \mathrm{Mg}, \mathrm{Si}$, $\mathrm{S}$, and $\mathrm{Fe}$. We recall that in the present calculations the chemical mixture of metals at the ZAMS is assumed to be scaled solar, according to the Grevesse \& Noels (1993) compilation. While the surface abundances of $\mathrm{C}$ and $\mathrm{O}$ may change in the course of the stellar evolution (mainly due to dredge-up episodes and $\mathrm{HBB}$ ), those of $\mathrm{Mg}, \mathrm{Si}, \mathrm{S}$, and $\mathrm{Fe}$ remain practically unaltered.

Following the results of non-equilibrium dust-formation calculations carried out by Ferrarotti \& Gail (2006), it is useful to define two critical carbon abundances, namely $Y_{\mathrm{C}, 1}=Y_{\mathrm{O}}-2 Y_{\mathrm{Si}}$ and $Y_{\mathrm{C}, 2}=Y_{\mathrm{O}}-Y_{\mathrm{Si}}+Y_{\mathrm{S}}$, where $Y=X / A$ is the abundance in mol $\mathrm{g}^{-1}$. They define sharp boundaries between three different domains of dust compounds, as described in the following.

Adopting the solar $\mathrm{O}$ abundance of Grevesse \& Noels (1993), and under the assumption that the surface abundance of $\mathrm{O}$ is not affected during the evolution, then $Y_{\mathrm{C}, 1}$ and $Y_{\mathrm{C}, 2}$ define

7 Following Ferrarotti \& Gail (2006), with seed or key element we denote the least abundant element among those required to form the dust species under consideration; e.g., Si is the seed element of the silicate compounds in O-rich stars. two critical $\mathrm{C} / \mathrm{O}$ ratios, namely $(\mathrm{C} / \mathrm{O})_{1}=0.90$ and $(\mathrm{C} / \mathrm{O})_{2}=$ 0.97 . The invariance of $\mathrm{O}$ is a common assumption, since standard predictions of detailed AGB nucleosynthesis indicate that the material dredged up at thermal pulses comprises only a small amount of oxygen, i.e., $X_{\mathrm{O}} \sim 0.5-4 \%$ (Boothroyd \& Sackmann 1988; Izzard et al. 2004). However, one should consider that this result is highly dependent on the treatment of convective boundaries (see e.g., Herwig 2000, for high O inter-shell abundances); in addition the surface abundance of $\mathrm{O}$ may be even depleted as a consequence of efficient HBB in low-metallicity high-mass AGB stars (Ventura et al. 2002).

Since in our calculations the oxygen variations are negligible in most cases, we adopt the initial $\mathrm{O}$ abundance to define three main regimes of dust composition as a function of $(\mathrm{C} / \mathrm{O})_{1}$ and $(\mathrm{C} / \mathrm{O})_{2}$, which broadly correspond to the three main spectral classes of AGB stars (M, S, C). Details are given in Sects. 4.3.1 to 4.3.3.

When applied to our isochrones, the formalism outlined above produces $\Psi$ values typically up to $0.004,0.002,0.0001$ for O-rich stars, and up to $0.01,0.02,0.02$ for C-rich stars, with $Z=0.019,0.008,0.001$, respectively. These are maximum predicted values, and they are not meant to be representative of AGB stars at varying $Z$. Detailed population synthesis models are needed to explore this issue, which is postponed to future analysis.

Regardless, from comparing the upper values given above we derive some interesting trends. While for O-rich stars we find a direct proportionality between $\Psi$ and $Z$, for C-rich stars the relation becomes more complex and non-monotonic. In the case of O-rich envelopes the relation $\psi \propto Z$ is explained by considering that the maximum amount of dust that can be formed is controlled by the abundances of the seed elements (i.e., $\mathrm{Si}, \mathrm{Fe}$ ), which scale with the initial metallicity.

In the case of C-rich envelopes one key parameter in the formation of dust is the excess of carbon with respect to oxygen, and this quantity does not trace the initial metallicity as it is crucially affected by the third dredge-up and HBB during the TP-AGB phase. It follows that the large $\Psi$ values predicted for $\mathrm{C}$-rich stars even at low $Z$ reflect their relatively high $\mathrm{C} / \mathrm{O}$ ratios as predicted by our TP-AGB models at decreasing metallicity.

It is worth noticing that these theoretical expectations are in line with the observational evidence presented in van Loon (2000) and Marshall et al. (2004) for the proportionality of the 
dust-to-gas ratio on $Z$ in oxygen-rich stars; and van Loon et al. (1999) for the enhanced abundance of free carbon available for molecule and dust formation in metal-poor carbon stars. More observational data on this issue has been recently provided by e.g., Speck et al. 2006; Thompson et al. 2006; Sloan et al. 2006; Zijlstra et al. 2006; Lagadec et al. 2007; Matsuura et al. 2007.

\subsubsection{Stars with $\mathrm{C} / \mathrm{O}<0.90$}

In this interval, there are sufficient oxygen atoms to allow the formation of the silicate-type dust. These conditions roughly correspond to the domain of $\mathrm{M}$ stars. Two main dust contributions are considered:

$\frac{\mathrm{d} M_{\text {dust }}}{\mathrm{d} t}=\frac{\mathrm{d} M_{\text {sil,M }}}{\mathrm{d} t}+\frac{\mathrm{d} M_{\text {iro,M }}}{\mathrm{d} t}$

that correspond to silicate dust and iron dust, respectively. These can be defined as

$$
\begin{aligned}
& \frac{\mathrm{d} M_{\text {sil,M }}}{\mathrm{d} t}=\dot{M} X_{\mathrm{Si}} \frac{A_{\text {sil }}}{A_{\mathrm{Si}}} f_{\text {sil }} \\
& \frac{\mathrm{d} M_{\text {iro,M }}}{\mathrm{d} t}=\dot{M} X_{\mathrm{Fe}} \frac{A_{\text {iro }}}{A_{\mathrm{Fe}}} f_{\text {iro }}
\end{aligned}
$$

where $A_{\mathrm{Si}}\left(A_{\mathrm{Fe}}\right)$ is the atomic weight of $\mathrm{Si}(\mathrm{Fe}), A_{\mathrm{Si}}\left(A_{\text {iro }}\right)$ is the effective molecular weight and $f_{\text {sil }}\left(f_{\text {iro }}\right)$ is the condensation degree of the silicate (iron) dust.

The condensation degree of silicate dust, $f_{\text {sil }}$ accounts for the possible contributions of olivine-type dust, pyroxene-type dust, and quartz-type dust:

$f_{\text {sil }}=f_{\text {ol }}+f_{\text {py }}+f_{\text {qu }}$

with clear meaning of the symbols. The effective molecular weight of the silicate dust mixture is expressed

$A_{\text {sil }}=\left(f_{\mathrm{ol}} A_{\mathrm{ol}}+f_{\mathrm{py}} A_{\mathrm{py}}+f_{\mathrm{qu}} A_{\mathrm{qu}}\right) / f_{\mathrm{sil}}$.

From the analysis of Ferrarotti \& Gail (2001), the amount of silicate dust in form of olivine, pyroxene, and quartz depends critically on the $Y_{\mathrm{Mg}} / Y_{\mathrm{Si}}$ ratio, which is $\sim 1.07$ according to the chemical mixture adopted in this work (Grevesse \& Noels 1993). At this value the non-equilibrium dust condensation calculations by Ferrarotti \& Gail (2001) predict that the dust mixture is mainly composed of olivine and pyroxene, with a small fraction of quartz. Following the results for an M-star model with $\dot{M}=10^{-5} M_{\odot} \mathrm{yr}^{-1}$ and $Y_{\mathrm{Mg}} / Y_{\mathrm{Si}} \simeq 1.07$, the condensation degrees are assumed to obey the ratios $f_{\mathrm{ol}} / f_{\mathrm{py}}=4$ and $f_{\mathrm{ol}} / f_{\mathrm{qu}}=22$.

Finally, $f_{\text {sil }}$ and $f_{\text {iro }}$ are evaluated with the analytic fits provided by Ferrarotti (2003):

$$
\begin{aligned}
& f_{\text {sil }}=0.8 \frac{\dot{M}_{-6}}{\dot{M}_{-6}+5} \sqrt{\frac{Y_{\mathrm{C}, 1}-Y_{\mathrm{C}}}{Y_{\mathrm{C}, 1}}} \\
& f_{\text {iro }}=0.5 \frac{\dot{M}_{-6}}{\dot{M}_{-6}+5} .
\end{aligned}
$$

\subsubsection{Stars with $0.90 \leq \mathrm{C} / \mathrm{O}<0.97$}

In this interval a small amount of silicates can be produced because of the deficit in $\mathrm{O}$ and the dust composition is irondominated. These conditions may roughly include the class of $\mathrm{S}$ stars. Two main dust contributions are considered:

$$
\frac{\mathrm{d} M_{\text {dust }}}{\mathrm{d} t}=\frac{\mathrm{d} M_{\text {sil, } \mathrm{M}}}{\mathrm{d} t}+\frac{\mathrm{d} M_{\text {iro,M }}}{\mathrm{d} t}
$$

which correspond to silicate dust and iron dust, respectively. These can be defined as

$$
\begin{aligned}
& \frac{\mathrm{d} M_{\text {sil,M }}}{\mathrm{d} t}=\dot{M} X_{\mathrm{Si}} \frac{A_{\text {sil }}}{A_{\mathrm{Si}}} f_{\text {sil }} \\
& \frac{\mathrm{d} M_{\text {iro,M }}}{\mathrm{d} t}=\dot{M} X_{\mathrm{Fe}} \frac{A_{\text {iro }}}{A_{\mathrm{Fe}}} f_{\text {iro }}
\end{aligned}
$$

with the same meaning of the symbols as in Sect. 4.3.1.

Following Ferrarotti (2003) the condensation degrees are approximated with

$f_{\text {sil }, \mathrm{S}}=0.1 \frac{\dot{M}_{-6}}{\dot{M}_{-6}+5}$
$f_{\text {iro }, \mathrm{S}}=0.5 \frac{\dot{M}_{-6}}{\dot{M}_{-6}+5}$.

We note that for given $\dot{M}$ the total condensation degree of silicate dust is lower than in the case of M stars (because of the lower abundance of available $\mathrm{O}$ ). The relative ratios between $f_{\mathrm{ol}}, f_{\mathrm{py}}$, and $f_{\text {qu }}$ are assumed the same as those for M-stars, which is a reasonable assumption (see Ferrarotti \& Gail 2002).

\subsubsection{Stars with $\mathrm{C} / \mathrm{O} \geq 0.97$}

In this interval, the dust mixture becomes carbon-dominated. These conditions apply to the class of C stars. Two dust contributions are included:

$\frac{\mathrm{d} M_{\mathrm{dust}}}{\mathrm{d} t}=\frac{\mathrm{d} M_{\mathrm{sic}, \mathrm{C}}}{\mathrm{d} t}+\frac{\mathrm{d} M_{\mathrm{car}, \mathrm{C}}}{\mathrm{d} t}$

which correspond to silicon carbide dust and solid carbon dust, respectively. These can be defined as

$$
\begin{aligned}
& \frac{\mathrm{d} M_{\text {sic }, \mathrm{C}}}{\mathrm{d} t}=\dot{M} X_{\mathrm{Si}} \frac{A_{\text {sic }}}{A_{\mathrm{Si}}} f_{\text {sic }} \\
& \frac{\mathrm{d} M_{\mathrm{car}, \mathrm{C}}}{\mathrm{d} t}=\dot{M} X_{\mathrm{C}} f_{\mathrm{car}} .
\end{aligned}
$$

$X_{\mathrm{Si}}$, and $X_{\mathrm{C}}$ represent the element abundances (in mass fraction), which are given by the initial values on the main sequence for $\mathrm{Fe}$ and $\mathrm{Si}$, while the current atmospheric value is adopted for $\mathrm{C}$. The quantities $A_{\mathrm{Si}}$ and $A_{\text {sic }}$ are the atomic weight of $\mathrm{Si}$, and the effective molecular weight of the silicate dust mixture, respectively.

Finally, $f_{\text {sic }}$, and $f_{\text {car }}$ denote the degree of condensation of the elements $\mathrm{Si}$ and $\mathrm{C}$ in the corresponding dust species. Following Ferrarotti (2003) they are evaluated with

$$
\begin{aligned}
& f_{\text {sic }}=0.5 \frac{\dot{M}_{-6}}{\dot{M}_{-6}+5} \\
& f_{\text {car }}=0.5 \frac{\dot{M}_{-6}}{\dot{M}_{-6}+5}\left(\frac{\mathrm{C}}{\mathrm{O}}-1\right) .
\end{aligned}
$$

\subsection{Radiative transfer calculations}

We provide bolometric corrections from two independent RT calculations, which are updates and extensions of those originally described by B98 and G06. In both cases the models have been calculated with 1-dimensional codes that solve the RT equation and the thermal balance equation in a selfconsistent way (see Granato \& Danese 1994; Groenewegen 1993; and Groenewegen 1995).

Although they differ in several minor details related to the structure of the original codes - as described in Sects. 4.4.1 
and 4.4.2 - B98 and G06 calculations produce very similar results, proving the robustness of both RT models. For instance, whereas B98 tabulate the complete output $F_{\lambda}$ spectra with a regular spacing in $\log \lambda$ (here set to be $\Delta \log \lambda=0.04 \mathrm{dex}$ ), G06 code directly provides the integrated $F_{\lambda_{\text {eff }}}$ for a given set of input pass-bands. In fact, we have estimated that the differences in $\Delta \mathrm{BC}_{\lambda_{\mathrm{eff}}}$ coming from these two different approaches, for the broadband filters we are using, are of the order of just $\sim 0.05$ mag for $\tau(1 \mu \mathrm{m})=1$. Considering the overall uncertainties, these differences are small enough to be safely ignored.

Instead, much more important are the differences associated with the assumed dust compositions, and the coverage of stellar parameters, as detailed below.

\subsubsection{Extended G06 calculations}

The dust models that are incorporated in the isochrones are an extension of the work described in G06. We have calculated the models for (arbitrary) values of $L=3000 L_{\odot}, d=8.5 \mathrm{kpc}$, $v_{\text {exp }}=10 \mathrm{~km} \mathrm{~s}^{-1}, \Psi=0.005$. In G06, only few effective temperatures were considered. Here we calculated models for spectral types M0, 1, .., $10\left(T_{\text {eff }}=3850,3750,3650,3550,3490,3397\right.$, 3297, 3129, 2890, 2667, $2500 \mathrm{~K}$ ) from Fluks et al. (1994) for O-rich stars, and $T_{\text {eff }}=3400,3200,3000,2800,2650 \mathrm{~K}$ from Loidl et al. (2001) for C-rich stars (with a C/O ratio of 1.1). The $\mathrm{O}$ - and C-stellar input spectra are for solar metallicities.

Several types of dust are considered (see G06 for a discussion). For O-rich stars, (1) $100 \%$ Aluminium Oxide (AlOx; amorphous porous $\mathrm{Al}_{2} \mathrm{O}_{3}$ ), with optical constants from Begemann et al. (1997), (2) a combination of 60\% AlOx and $40 \%$ Silicate (optical constants from David \& Pégourié 1995), (3) $100 \%$ silicate. A condensation temperature of $T_{\mathrm{c}}=1500 \mathrm{~K}$ was assumed in the first two cases, $1000 \mathrm{~K}$ for the pure silicate dust.

For C-stars, we consider either a combination of $85 \%$ Amorphous Carbon (AMC) and 15\% Silicon Carbide (SiC) with optical constants from, respectively, Rouleau \& Martin (1991) for the AC1 species and $\alpha$-SiC from Pégourié (1988), and assuming a $T_{\mathrm{c}}$ of $1200 \mathrm{~K}$, or $100 \%$ AMC with a $T_{\mathrm{c}}$ of $1000 \mathrm{~K}$.

The results of such computations consist in the apparent stellar magnitudes for several values of $\dot{M}$ (and hence $\tau$ ), ranging from $10^{-10}$ to $4 \times 10^{-5} M_{\odot} \mathrm{yr}^{-1}$, and for each spectral type and filter under consideration. Over this range of mass loss $\tau$ increases up to $\sim 3$ for AMC (at $11.33 \mu \mathrm{m}$ ) and $\sim 45$ for AlOx (at $11.75 \mu \mathrm{m}$ ). We considred a total of 90 filters in these specific computations. We converted these magnitude tables into $\triangle \mathrm{BC}(\tau)$ values that directly give the difference between the magnitudes of non-dusty $(\tau=0)$ and dusty stars ${ }^{8}$.

\subsubsection{B98 calculations}

In B98 for each dust type (either O-rich or C-rich) the reference grid of AGB dusty envelopes consists of models calculated for $L=10000, T_{\text {eff }}=3000 \mathrm{~K}$, while varying the optical depth $\tau(1 \mu \mathrm{m})$ from $5 \times 10^{-4}$ to 200 in 50 equal logarithmic steps. The condensation temperature is assumed $T_{\mathrm{c}}=1000 \mathrm{~K}$ for O-rich envelopes, and $T_{\mathrm{c}}=1500 \mathrm{~K}$ for $\mathrm{C}$-rich envelopes. Additional tests have shown that, for the adopted dust mixtures, the resulting spectral shapes are little affected by changes in $L$ and $T_{\text {eff }}$ within the typical ranges for AGB stars, for fixed values of $\tau(1 \mu \mathrm{m})$.

\footnotetext{
8 The use of $\triangle \mathrm{BC}(\tau)$ ensures that any residual difference in the zero-points between the G06 magnitudes, and those used in our new isochrones, will be erased.
}

The radiative transfer of the photospheric stellar spectrum through the dust is computed with the Granato \& Danese (1994) code (originally built for AGN tori), assuming spherical symmetry and the radial dust density distribution decreasing as $r^{-2}$. The adopted dust grain model is that of Rowan-Robinson et al. $(1986)^{9}$, which considers six different kinds of grains: AMC of radius $a=0.1 \mu \mathrm{m}$ and graphites of 0.03 and $0.01 \mu \mathrm{m}$ for C-rich mixtures, and amorphous silicate of $a=0.1 \mu \mathrm{m}$ and silicates of 0.03 and $0.01 \mu \mathrm{m}$ for O-rich mixtures.

Although we updated in several aspects the B98 model, in particular in the dust grain properties, the detailed exploration with Spitzer data of these updates is still underway and will be presented in a forthcoming paper. Therefore here we adopt the already well-established B98 model in its original form. The dust composition we consider is carbon for C-type mass-losing stars, and silicates for M-type ones.

The relation between $\tau(1 \mu \mathrm{m})$ and the mass loss is given by Eq. (4) with $A_{\mathrm{d}}=1.5 \times 10^{11}$ for silicates and $A_{\mathrm{d}}=6.6 \times 10^{11}$ for the carbonaceous mix (with $v_{\text {exp }}$ in $\mathrm{km} \mathrm{s}^{-} 1, L$ in solar units). The quantity $A_{\mathrm{d}}$ is slightly affected by the spectrum of the illuminating star. However, B98 have checked that the errors in $\tau(1 \mu \mathrm{m})$ estimated from Eq. (4) with the above values of $A_{\mathrm{d}}$ are kept within $5 \%$ for spectra with $T_{\text {eff }}$ in the range $2500-4000 \mathrm{~K}$. This is a fair approximation because models with $\tau(1 \mu \mathrm{m})$ differing by less than $5-10 \%$ produce almost identical spectra.

\subsubsection{Overview of RT models}

Figure 3 illustrates the behaviour of $\triangle \mathrm{BC}$ as a function of wavelength, for the dust compositions considered in this work, and for the specific case of $\tau(1 \mu \mathrm{m})=1$ and $T_{\text {eff }}=3000 \mathrm{~K}$. We note that all curves agree very well in the case of O-rich mixtures, and differ significantly only for the C-rich mixtures at $\lambda>10 \mu \mathrm{m}$. These differences become higher for higher values of $\tau$, and can be ascribed to the use of different dust mixtures and spectral libraries.

In summary, we consider several possible choices of dust composition, namely: four for O-rich envelopes and three for Crich envelopes. Alternatively, we can ignore dust setting $\tau=0$. All these cases are allowed in our isochrone calculations.

\section{A preliminary comparison with observations}

Gi00 isochrones have been used extensively in the literature for the study of resolved stellar populations. Most of their use has regarded the optical photometry of stars in the most populated evolutionary phases like the main sequence, sub-giant branch, RGB, red clump and early-AGB. Now the addition of the detailed TP-AGB evolution together with the infrared photometry open new perspectives for the application of these new stellar models, which we briefly illustrate in the following via comparisons with Galactic Disc and Magellanic Cloud data.

Most of the comparisons are intended to illustrate the locus of our new isochrones in different diagrams, and do not consider crucial aspects of the host galaxies, such as their age-metallicity relations and star formation histories. A detailed comparison, between present models and the observed star counts in several parts of these diagrams, is left to future papers.

\footnotetext{
9 The dust model by Rowan-Robinson et al. (1986) is based on observations of circumstellar dust shells around late-type C- and M-type stars that appear to require absorption efficiencies decreasing as $\lambda^{-1}$ in the far-IR. This wavelength dependence is ascribed to grains with a disordered, damaged or amorphous structure. See the original paper for more details.
} 

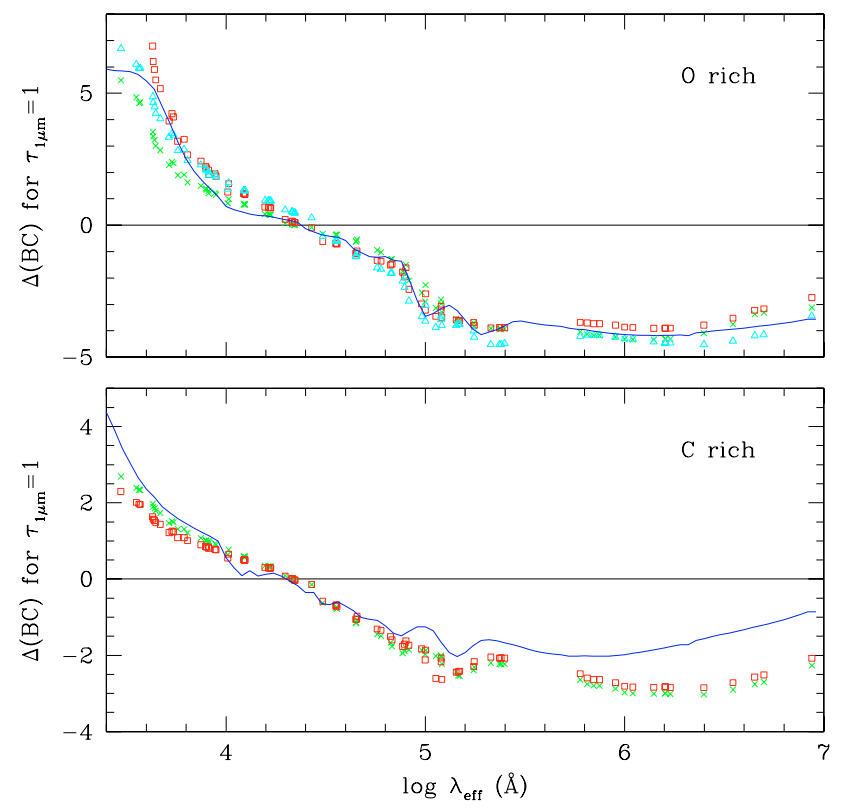

Fig. 3. The absorption/emission properties of the dust mixtures considered in this work, in the form of $\triangle \mathrm{BC}$ values and limited to the case of $\tau_{1 \mu \mathrm{m}}=1$ and input stellar spectra of $T_{\mathrm{eff}}=3000 \mathrm{~K}$. Top panel: for O-rich stars, the dust compositions are 100\% AlOx (crosses), 100\% silicates (triangles), $60 \% \mathrm{AlOx}+40 \%$ silicates, (squares), following the G06 approach, and silicates (continuous line) following B98. Bottom panel: for C-rich stars, the dust compositions are $100 \%$ AMC (crosses) and $85 \% \mathrm{AMC}+15 \% \mathrm{SiC}$ (squares), following the G06 approach, and graphites (continuous line) following B98.

\subsection{Expansion velocities of variable AGB stars}

As a first check of our models, we compare the predicted expansion velocities (Sect. 4.2) as a function of pulsation periods with observations of variable AGB stars in the Galactic Disc and Magellanic Clouds. The results, displayed in Fig. 4, are consistent with the empirical estimates.

A relevant remark should be made in respect to the O-rich stars. While for $P<500-600$ days a positive correlation between periods and expansion velocities seems to exist in the observed data, this trend does not proceed at longer periods, where a considerable scatter of $v_{\exp }$ is detected. It should be noticed that while reproducing the mean observed trend at shorter periods, the widely-used relation of Vassiliadis \& Wood (1993; solid line) fails to describe the data at longer periods, where $v_{\exp }$ is assumed to be constant $\left(15 \mathrm{~km} \mathrm{~s}^{-1}\right)$.

In the bottom panels of Fig. 4 we show the results of our calculations for a few selected isochrones with $Z=0.019$ and $Z=0.008$. Regardless of age all isochrones describe a similar non-monotonic evolution on the $v_{\exp }-P$ diagram. At increasing periods, first the expansion velocity increases, reaches a maximum and eventually declines. This bell-shaped behaviour is particularly well developed for the youngest isochrones (with ages of $\sim 1.6 \times 10^{8} \mathrm{yr}$ and $\sim 2 \times 10^{8} \mathrm{yr}$ ), which correspond to massive AGB stars (with initial masses of $4.5 M_{\odot}$ and $4.1 M_{\odot}$ ) and it appears to explain the existence of variables with high periods and relatively low expansion velocities.

With respect to this, it is also interesting to compare the two isochrones with $\log ($ age $/ y r)=8.3)$ and different metallicities $(Z=0.019$ and $Z=0.008)$. The one with $Z=0.008$ reaches, on average, lower $v_{\text {exp }}$. Moreover, the few OH/IR stars in the LMC for which $v_{\exp }$ has been measured (empty squares; Marshall et al. 2004) fall in correspondence with the declining branches of the

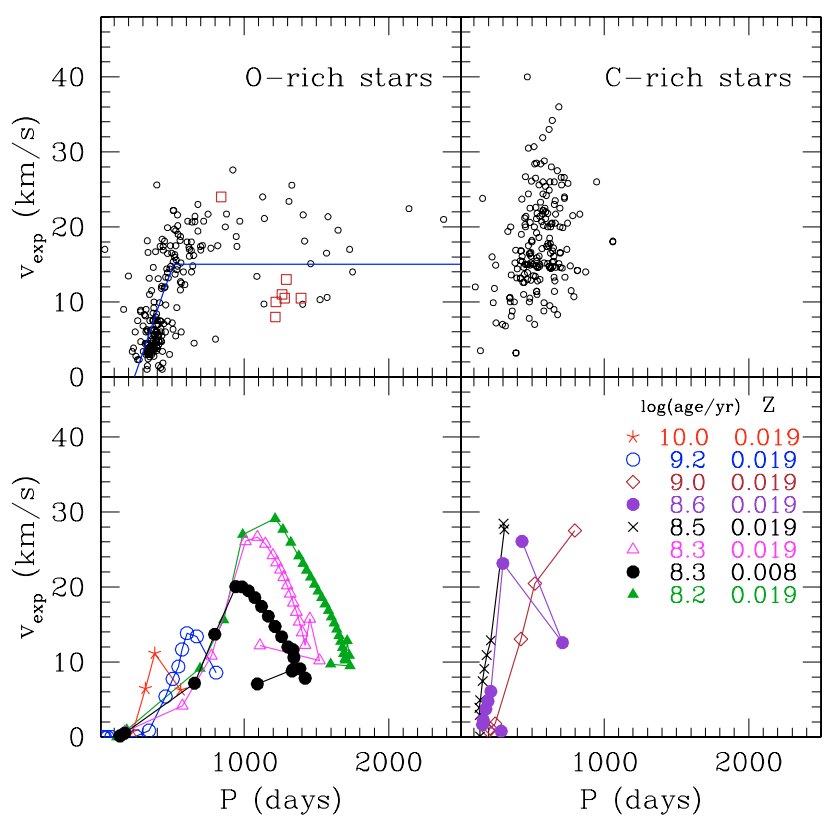

Fig. 4. Expansion velocities of circumstellar flows against pulsation periods of variable AGB stars, for both O-rich (left panels) and C-rich objects (right panels). Upper panels: galactic observations (empty circles) are taken from the compilations assembled by Groenewegen et al. (1998) for O-rich stars and Groenewegen et al. (2002) for C-rich stars. Observed data for LMC variables (Marshall et al. 2004) is also plotted (empty squares). The fit relation proposed by Vassiliadis \& Wood (1993) is shown by the solid line. Bottom panels: predicted expansion velocities for a few selected isochrones with $Z=0.019$ and $Z=0.008$. Ages are indicated in the figure. See the text for more details.

$v_{\text {exp }}$ curves. This fact suggests that the low expansion velocities of LMC OH/IR stars compared to those of Galactic OH/IR stars with the same $P$, may not reflect a pure effect of metallicity (i.e., a linear $Z$-dependence of the dust-to-gas ratio $\Psi$ such that the lower $Z$, the lower $v_{\text {exp }}$ ), as commonly invoked (see the discussion in Marshall et al. 2004); rather it may be the result of a more complex dependence of $v_{\exp }$ as a function of stellar and dust parameters $(L, \dot{M}, M, \Psi)$. We will perform a deep investigation of this issue in future work.

\subsection{The LMC and SMC near-infrared photometry}

TP-AGB stars become striking objects in galactic surveys conducted at pass-bands redder than $I$. The best available data for the AGB population in galaxies is likely the data obtained from the extensive near- and mid-infrared surveys of the Magellanic Clouds.

In Fig. 5, we plot the 2MASS $K_{\mathrm{S}}$ vs. $J-K_{\mathrm{S}}$ diagram of the inner LMC and SMC. The data are taken directly from the SAGE and $\mathrm{S}^{3} \mathrm{MC}$ catalogs (Blum et al. 2006; and Bolatto et al. 2007, respectively), and hence correspond to objects that have counterparts in the Spitzer pass-bands.

We start discussing the LMC case depicted in the left panel. The circular area of $\pi$ sqrdeg centered on the LMC bar at $\alpha_{2000}=$ $5^{\mathrm{h}} 23 \mathrm{~m} .5, \delta_{2000}=-69^{\circ} 45^{\prime}$, is large enough to include thousands of AGB stars brighter than the TRGB, and small enough to somewhat limit the presence of features that are not due to the LMC. These latter features are, however, still evident in the plot, and consist of (1) the vertical fingers in the top left part of the CMD, at $K_{\mathrm{s}}<11 \mathrm{mag}$ and $J-K_{\mathrm{s}}<1.0 \mathrm{mag}$, caused by foreground stars in the Milky Way disc and halo, and (2) the plume of objects at 

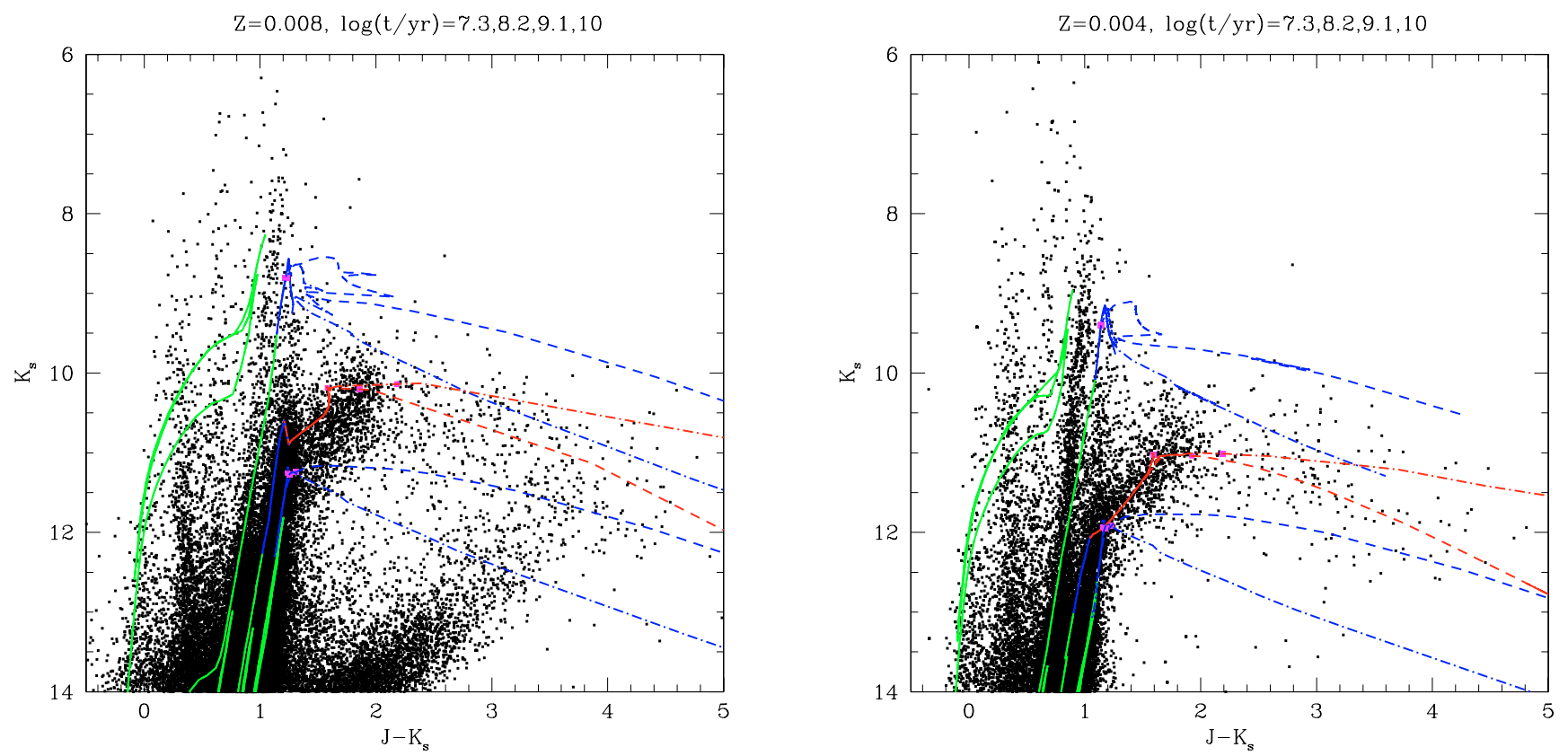

Fig. 5. The 2 MASS $K_{\mathrm{s}}$ vs. $J-K_{\mathrm{s}}$ diagram for the inner LMC (left panel) and for the SMC (right). Over-imposed are isochrones with $Z=0.008$ shifted by a distance modulus of 18.5 , and with $Z=0.004$ shifted by 19.0 , for the LMC and SMC cases, respectively. The isochrones are drawn in different colours according to their evolutionary status: pre-TP-AGB stars (green), and TP-AGB stars O-rich (blue) and C-rich (red). The selected ages are: $\log (t / \mathrm{yr})=7.3$ (no AGB present), 8.2 (well-developed O-rich TP-AGB), 9.1 (well-developed C-rich TP-AGB), and 10.0 (short O-rich TP-AGB), for 3 different choices of dust-obscuration: the no-dust case (continuous lines), cf. Bressan et al. (1998; dot-dashed lines) and cf. Groenewegen (2006; the $0.85 \mathrm{AMC}+0.15 \mathrm{SiC}$ mixture for $\mathrm{C}$-stars, and $0.6 \mathrm{AlOx}+0.4$ silicate for O-rich stars; dashed lines). In order to limit confusion in this plot, the isochrones are drawn up to their point of maximum mass-loss rate on the TP-AGB, i.e., ignoring their final post-AGB stages in which the isochrones cross back to the blue part of the diagram. Moreover, a magenta dot mark points where the mass loss increases above $10^{-6} M_{\odot} \mathrm{yr}^{-1}$; above this indicative limit the stellar lifetimes are necessarily short, and few stars are expected to occupy isochrone sections to the red of these points.

the bottom right part of the CMD, roughly at $K_{\mathrm{s}}>12$ mag vs. $J-K_{\mathrm{s}}>1.6 \mathrm{mag}$, which largely consists of background galaxies (see Nikolaev \& Weinberg 2000, his Region L). Outside of these two CMD regions, the bulk of objects indeed consists of stars located in the LMC.

On this diagram, we over-plot our isochrones for the initial metallicity $Z=0.008$ (which are adequate to describe the youngest LMC populations) and at four selected ages:

- At $\log (t / \mathrm{yr})=7.3$, AGB stars are not present and the most prominent near-IR stars are red super-giants burning He in their cores. These stars draw a diagonal line that coincides with sequence $\mathrm{H}$ of Nikolaev \& Weinberg (2000; their Fig. 3).

- At $\log (t / \mathrm{yr})=8.2$, the TP-AGB phase is well developed and populated by luminous $\mathrm{O}$-rich stars undergoing HBB. These stars draw a second diagonal sequence in the CMD, corresponding to sequence $\mathrm{G}$ of Nikolaev \& Weinberg (2000) and joining their sequence $\mathrm{F}$ at fainter magnitudes populated by early-AGB stars. These features are also well described by our new isochrones.

- At $\log (t / \mathrm{yr})=9.1$, HBB does not occur and the TP-AGB phase is dominated by a well-populated red tail of C-rich stars (sequence $J$ in Nikolaev \& Weinberg 2000), which departs from the O-rich sequence at $K_{\mathrm{s}}=10.3 \mathrm{mag}$, $J-K_{\mathrm{s}}=1.2 \mathrm{mag}$ and reaches $J-K_{\mathrm{s}}$ as red as $2.0 \mathrm{mag}$. This feature is overall well described by the $\log (t / \mathrm{yr})=9.1$ isochrones without dust, apart from the fact that in this case the red tail just reaches $J-K_{\mathrm{s}}=1.6 \mathrm{mag}$, which is the colour of all C-star spectra cooler than $\sim 3000 \mathrm{~K}$ in the Loidl et al. (2001) library.
- At $\log (t / \mathrm{yr})=10.0$, the third dredge-up does not occur, and the only TP-AGB stars are O-rich. They correspond to sequence F in Nikolaev \& Weinberg (2000), located right above the TRGB.

The above items mainly describe the behaviour of the isochrones at the regime of weak mass-loss, in which colours and magnitudes are affected by circumstellar dust at the level of just $\lesssim 0.2 \mathrm{mag}$. What happens in the regime of high mass-loss rates (say $|\dot{M}|>10^{-6} M_{\odot} \mathrm{yr}^{-1}$ ) is also well evident in the figure, when we look at the isochrones with dust obscuration: the upper fraction of their TP-AGB phase departs to very red colours at increasing $K_{\mathrm{s}}$-band magnitudes, reaching $J-K_{\mathrm{s}}>4$ mag. The location and slope of this extended red tail (sequence $\mathrm{K}$ in Nikolaev \& Weinberg 2000) is also well described by our isochrones, and especially by the $\log (t / \mathrm{yr})=9.1$ one which runs over the most densely populated part of this extended red tail.

The left panel of Fig. 6 details the region of the $K_{\mathrm{s}}$ vs. $J-K_{\mathrm{s}}$ diagram that includes the bulk of the TP-AGB stars. In addition to the 2MASS data for the inner LMC, we plot the Groenewegen (2004) database of LMC stars spectroscopically classified as being of types $\mathrm{M}$ and $\mathrm{C}$. The latter are LPVs selected from the OGLE database for the entire LMC. Although they are not distributed evenly over the CMD, they clearly illustrate that the bulk of M- (dark-green) and C-type (orange) stars are well separated in the CMD; in particular, they show the striking red tail of $\mathrm{C}$ stars extending from $J-K_{\mathrm{s}} \sim 1.3$ mag up to $J-K_{\mathrm{s}} \sim 2.0 \mathrm{mag}$, and the confinement of $\mathrm{M}$ stars at the bluest colours $\left(J-K_{\mathrm{s}} \lesssim 1.3 \mathrm{mag}\right)$.

Then, the figure evidences how the new isochrones reproduce the general appearance of the data, and in particular the 

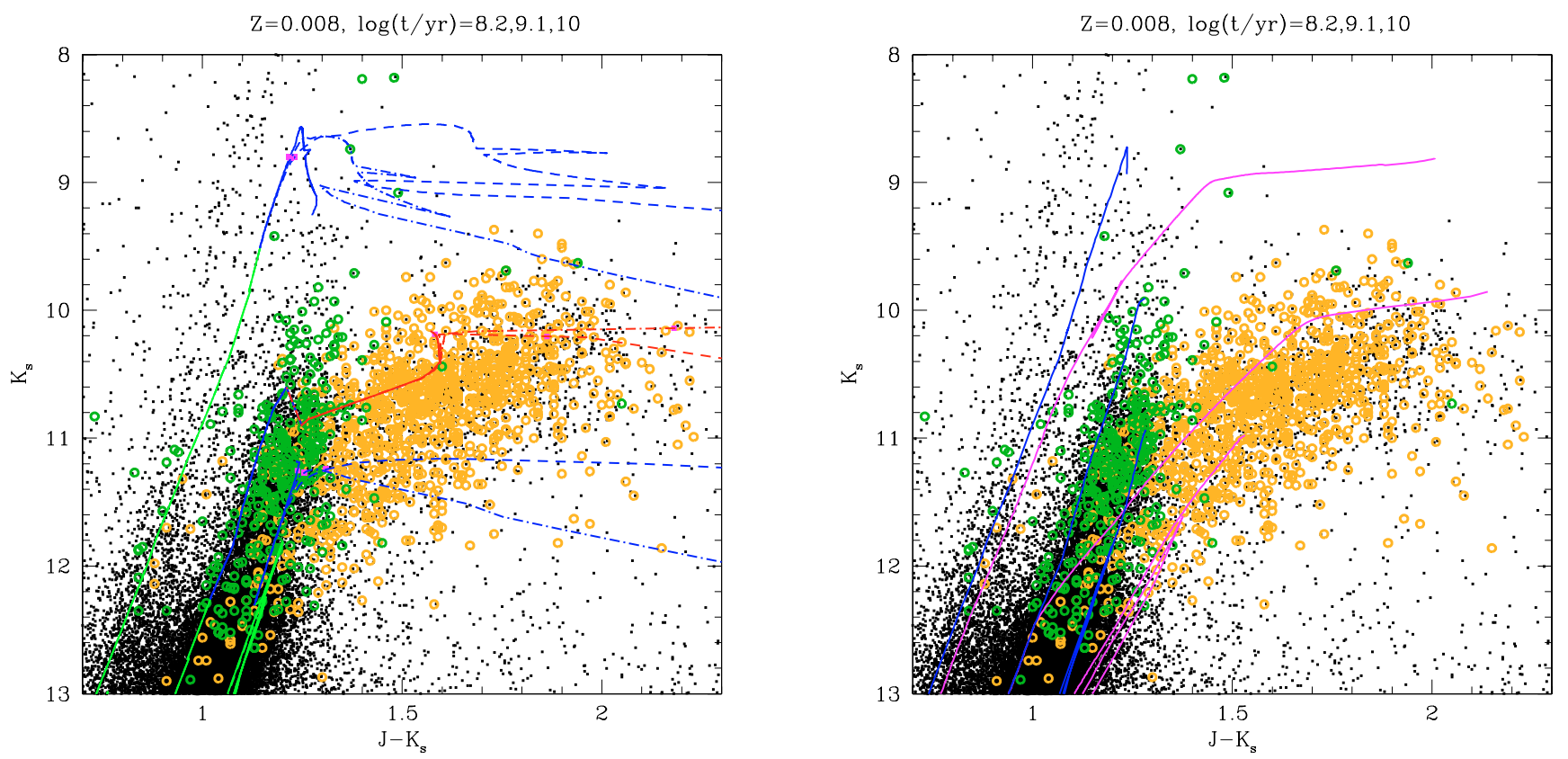

Fig. 6. Left panel: the 2MASS $K_{\mathrm{s}}$ vs. $J-K_{\mathrm{S}}$ diagram for the LMC, now detailing the CMD region that corresponds to the bulk of TP-AGB stars, and limiting the isochrones to the ages of $\log (t / \mathrm{yr})=8.2,9.1$, and 10.0. Additional LMC data are also plotted: the small circles are LPVs in the LMC that have been spectroscopically classified as being of type $\mathrm{M}$ (dark-green) or C (orange), according to the data compilation from Groenewegen (2004). The meaning of the different lines along the isochrones are the same as in Fig. 5. The right panel shows the same data as compared to Gi00 (blue lines) and Cordier et al. (2007; magenta lines). Notice that these latter sets of isochrones do not contain a C-star phase.

red tail of $\mathrm{C}$ stars which is well described by the $\log (t / \mathrm{yr})=$ 9.1 isochrone. It is also remarkable how well the isochrones predict the neat separation between O-rich (M-type) and C-rich (C-type) stars in the diagram. In the $\log (t / \mathrm{yr})=9.1$ isochrone, the red tail reaches $J-K_{\mathrm{s}} \sim 1.6$ even in absence of circumstellar dust. Dust obscuration in stars with $|\dot{M}| \lesssim 10^{-6} M_{\odot} \mathrm{yr}^{-1}$ provides an additional $\Delta\left(J-K_{\mathrm{s}}\right) \sim 0.2-0.3 \mathrm{mag}$ that improves the comparison at the reddest part of the red tail, at $J-K_{\mathrm{s}}$ between 1.6 and $2.0 \mathrm{mag}$. As thoroughly discussed in Marigo et al. (2003a), the main physical effect driving the appearance of the red tail of $\mathrm{C}$ stars are the cool $T_{\text {eff }}$ caused by changes in molecular opacities as the third dredge-up events increase the photospheric $\mathrm{C} / \mathrm{O}$ ratio.

The right panel of Fig. 6 over-plots isochrones from Gi00 and Cordier et al. (2007) over the same data. These isochrone sets contain the complete TP-AGB phase, but computed in a rather crude way, i.e. without considering the third dredge-up events and HBB nucleosynthesis, and using $T_{\text {eff }}\left(L, M, M_{\text {core }}\right)$ relations derived from scaled-solar mixtures. These isochrones fail to reproduce the basic features indicated by $2 \mathrm{MASS}$ and spectroscopic data, although in different ways:

- the Gi00 isochrones describe well the locus of O-rich AGB stars, as expected for TP-AGB models that do not consider the third dredge-up process. These isochrones are confined to $J-K_{\mathrm{S}} \lesssim 1.3 \mathrm{mag}$, and do not cross the red tail of C stars between $J-K_{\mathrm{s}} \sim 1.3$ and $2.0 \mathrm{mag}$;

- the Cordier et al. (2007) isochrones, instead, produce a sort of red tail, reaching $J-K_{\mathrm{s}} \sim 2.1 \mathrm{mag}$, but they do this while still on an $\mathrm{O}$-rich phase, and for all ages younger than about 1 Gyr. This is completely at odds with observations, which show that only C-rich stars (with very few exceptions) populate the red tail at $J-K_{\mathrm{s}}>1.3 \mathrm{mag}$.
The reasons why Cordier et al. (2007) O-rich TP-AGB models do reach such red $J-K_{\mathrm{s}}$ colours - so as to mimic C-type stars (although they are not) - likely reside in the use of i) the Wagenhuber (1996) formula for deriving $T_{\mathrm{eff}}-$ which allows to reach extremely low values, i.e., as cool as $2400 \mathrm{~K}$ at the TP-AGB end - and ii) a colour- $T_{\text {eff }}$ relation derived from C-type stars (from Bergeat et al. 2001). These choices are highly questionable. First, independent integrations of model atmospheres (e.g., Marigo 2002) suggest that O-rich stars can hardly reach $T_{\text {eff }}$ values lower than $\simeq 3000 \mathrm{~K}$, even at solar metallicities ${ }^{10}$. Second, it has long been known that $(J-K)-T_{\text {eff }}$ relations for $\mathrm{C}$-stars cannot be applied to O-rich stars, and vice-versa, since these two kinds of stars have very different near-infrared spectra.

From the failure of both the G00 and Cordier et al. (2007) isochrone sets in describing a red tail made of C stars, it follows that such models are not suitable for the interpretation of nearinfrared photometry in the age interval in which $\mathrm{C}$ stars form. For LMC metallicities, this interval is quite ample, going from a few $10^{8}$ yr up to about 5 Gyr (see also Frogel et al. 1990).

Finally, the 2MASS $K_{\mathrm{S}}$ vs. $J-K_{\mathrm{S}}$ diagram for the area within the SMC covered by the $S^{3} \mathrm{MC}$ survey (right panel of Fig. 5) can be described in a similar way to the LMC diagram. The most obvious difference between the two panels is in the $\sim 0.5 \mathrm{mag}$ shift of all features coming from the LMC-SMC difference in distance, which we also took in consideration when plotting

\footnotetext{
${ }_{10}$ Note that the bulk of $\mathrm{M}$ giants in the Magellanic Clouds are of spectral types earlier than $\mathrm{M} \mathrm{4}$, and in general, much hotter than the typical $\mathrm{M}$ giants in the Milky Way. A handful of cool M giants and supergiants of spectral type later than M 6 is also known in the Magellanic Clouds (e.g., Groenewegen \& Blommaert 1998; van Loon et al. 1998, 2005). In order to explain them, and also the more common giants of spectral type later than M 8 observed in the solar neighbourhood (with $T_{\text {eff }}$ as small as $2400 \mathrm{~K}$, see Fluks et al. 1994), our atmosphere models require super-solar metallicities.
} 

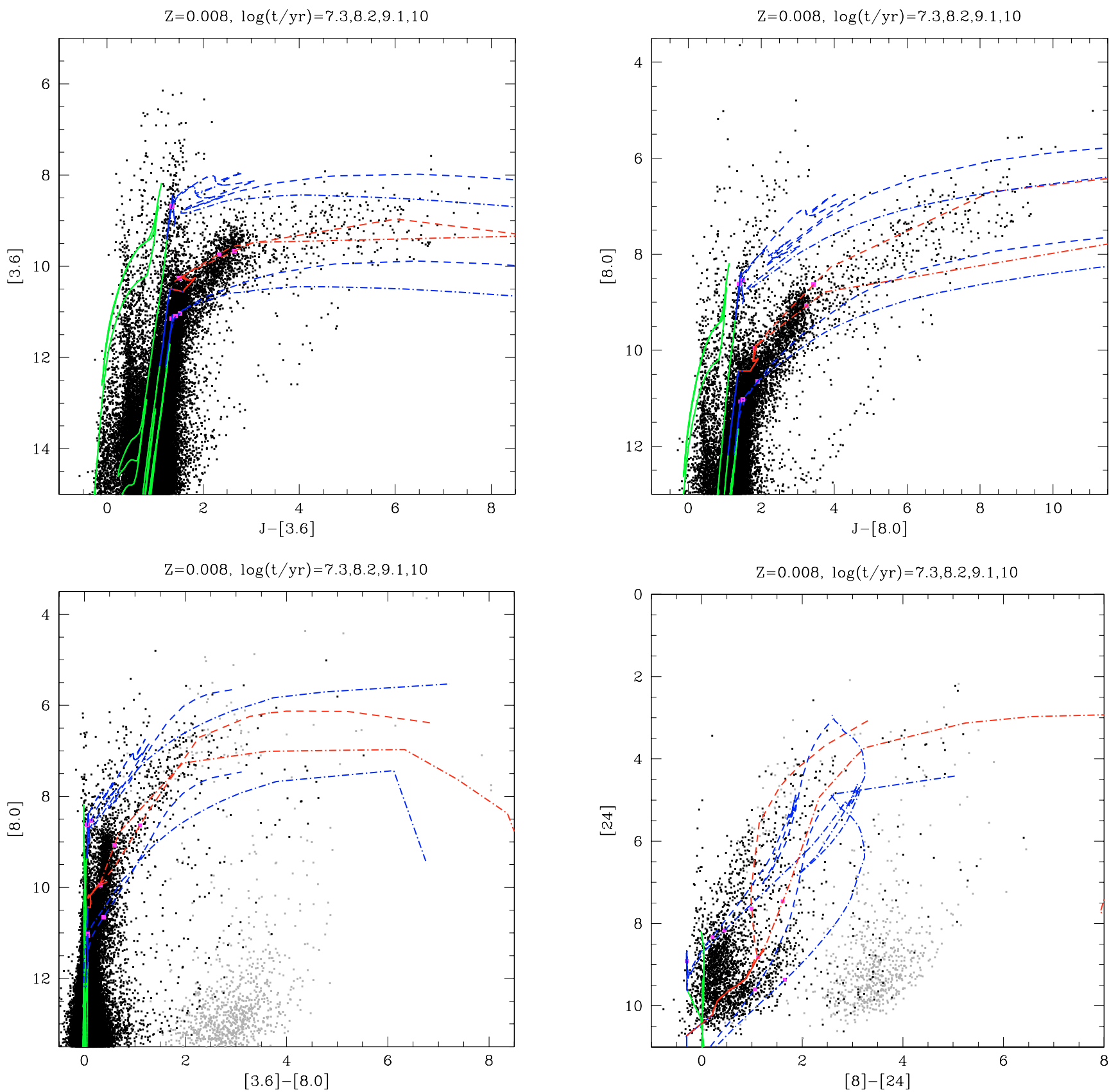

Fig. 7. A comparison between the new isochrones and the near- to mid-infrared data for the LMC. The isochrones are the same as plotted in the left panel of Fig. 5. The data (dots) come from the SAGE epoch 1 point source catalog (Blum et al. 2006) and combine IRAC+MIPS with 2MASS data inside a circle of 1 degree radius in the inner LMC. The panels show the 2MASS+IRAC [3.6] vs. $J-[3.6], 2 \mathrm{MASS}+\mathrm{IRAC}$ [8.0] vs. $J-$ [8.0], IRAC [8.0] vs. [3.6] - [8.0], and IRAC+MIPS [24] vs. [8.0]-[24] diagrams. The gray dots correspond to objects not detected by 2MASS, most of which are expected to correspond to background galaxies and young pre-main sequence stars, which are not discussed in this paper.

the isochrones. Also, due to the particular selection of data the SMC plot presents less contamination from background galaxies than the LMC plot.

\subsection{The $L M C$ and SMC mid-infrared photometry}

Figures 7 and 8 show a series of CMDs involving the near- and mid-IR photometry of the Magellanic Clouds, comparing the new isochrones with 2MASS+SAGE data for the inner LMC (Blum et al. 2006) and with $2 \mathrm{MASS}+\mathrm{S}^{3} \mathrm{MC}$ data for the SMC
(Bolatto et al. 2007) ${ }^{11}$. In all these plots, the effect of circumstellar dust becomes crucial in describing the observed photometry. The features present in these CMDs (except for the IRAC+MIPS [24] vs. [8.0]-[24] diagram, for which the data is poorer) are essentially the same as those present in the $K_{\mathrm{S}}$ vs. $J-K_{\mathrm{S}}$ diagram. In particular, all of them show contamination from foreground Milky Way stars in the form of a narrow vertical finger confined to the left part of the CMDs, and contamination from

11 The $\mathrm{S}^{3} \mathrm{MC}$ data, originally in flux units, have been converted to magnitudes using the zero-points from Cohen et al. (2003) for $J H K_{\mathrm{s}}$ and from Bolatto et al. (2007) for Spitzer pass-bands. 

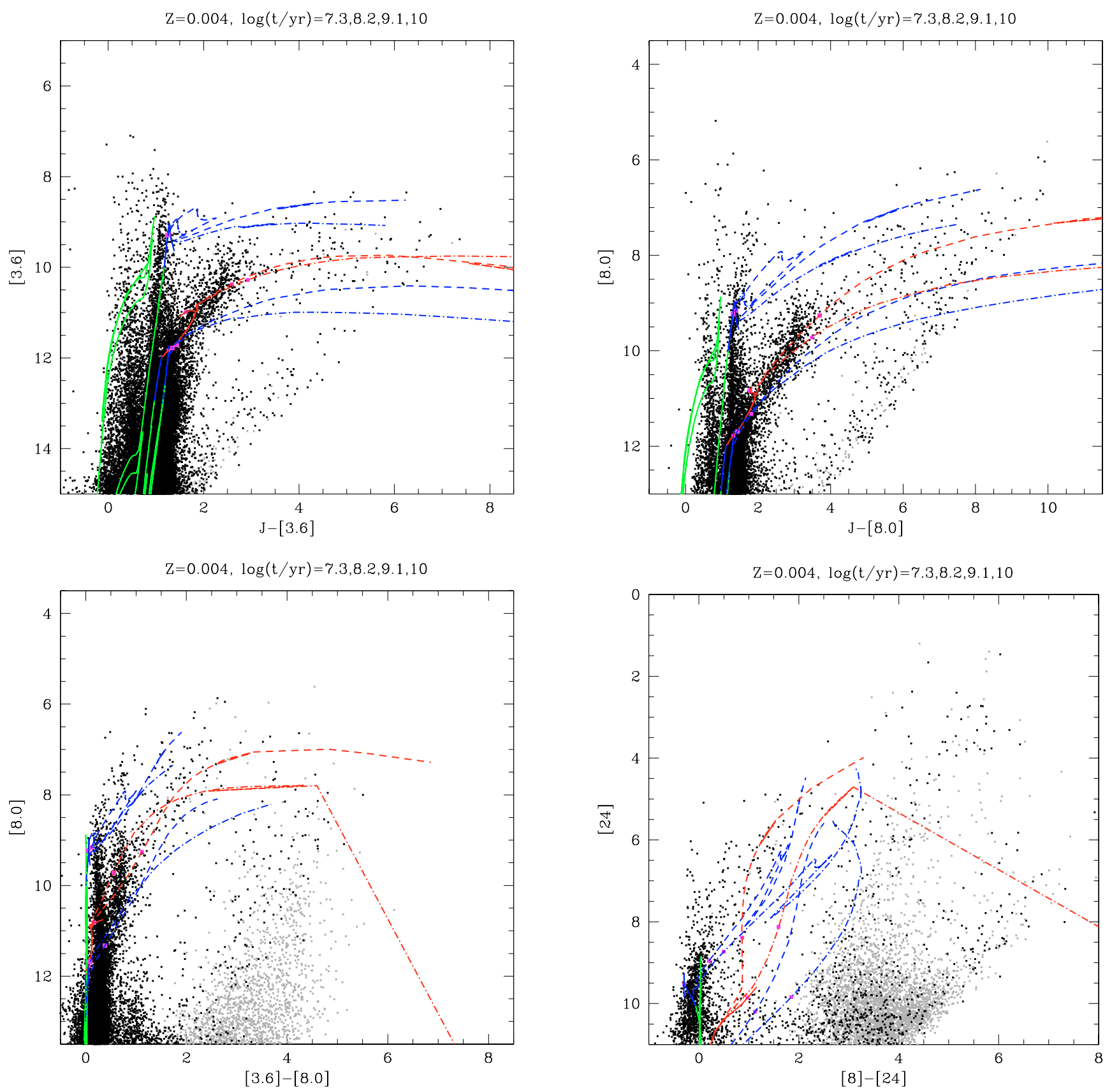

Fig. 8. As Fig. 7 but for the SMC. The data (dots) come from the $S^{3} \mathrm{MC}$ catalog (Bolatto et al. 2007). The isochrones are the same as in the right panel of Fig. 5.

background galaxies at their bottom-right corner. The gray dots correspond to objects not detected by 2MASS, most of which are expected to correspond to background galaxies and young pre-main sequence stars, which are not of interest in this paper.

The LMC population in these plots again shows O-rich sequences (core $\mathrm{He}$ burning red super-giants plus O-rich TP-AGBs) that progressively merge into an almost-vertical blue sequence when colours involving redder pass-bands are considered; plus an inclined $\mathrm{C}$-rich sequence that can be identified as the main body of the $J-K_{\mathrm{s}}<2.0 \mathrm{mag}$ red tail. From the extremity of this more populated red tail, a very extended and less populated plume departs, which corresponds to the stars with high mass-loss rates. Our isochrones reproduce well the position of these plumes in the several CMDs considered here, and moreover predict that they are populated by both O- and C-rich stars.

\subsection{Integrated broad-band colours}

Broadband colours of Magellanic Cloud clusters represent the basic observable against which any population synthesis model should be compared for testing its predicting performance. Figure 9 displays the results for two integrated colours, $V-K$ and $J-K$, which are expected to be significantly affected by the existence of TP-AGB stars. By looking at Fig. 9 (top panels), we actually see that the observed data for both $V-K$ and $J-K$ colours show a rising trend starting from $t \sim 10^{8}$ yr toward older ages, which is currently well explained by our current 

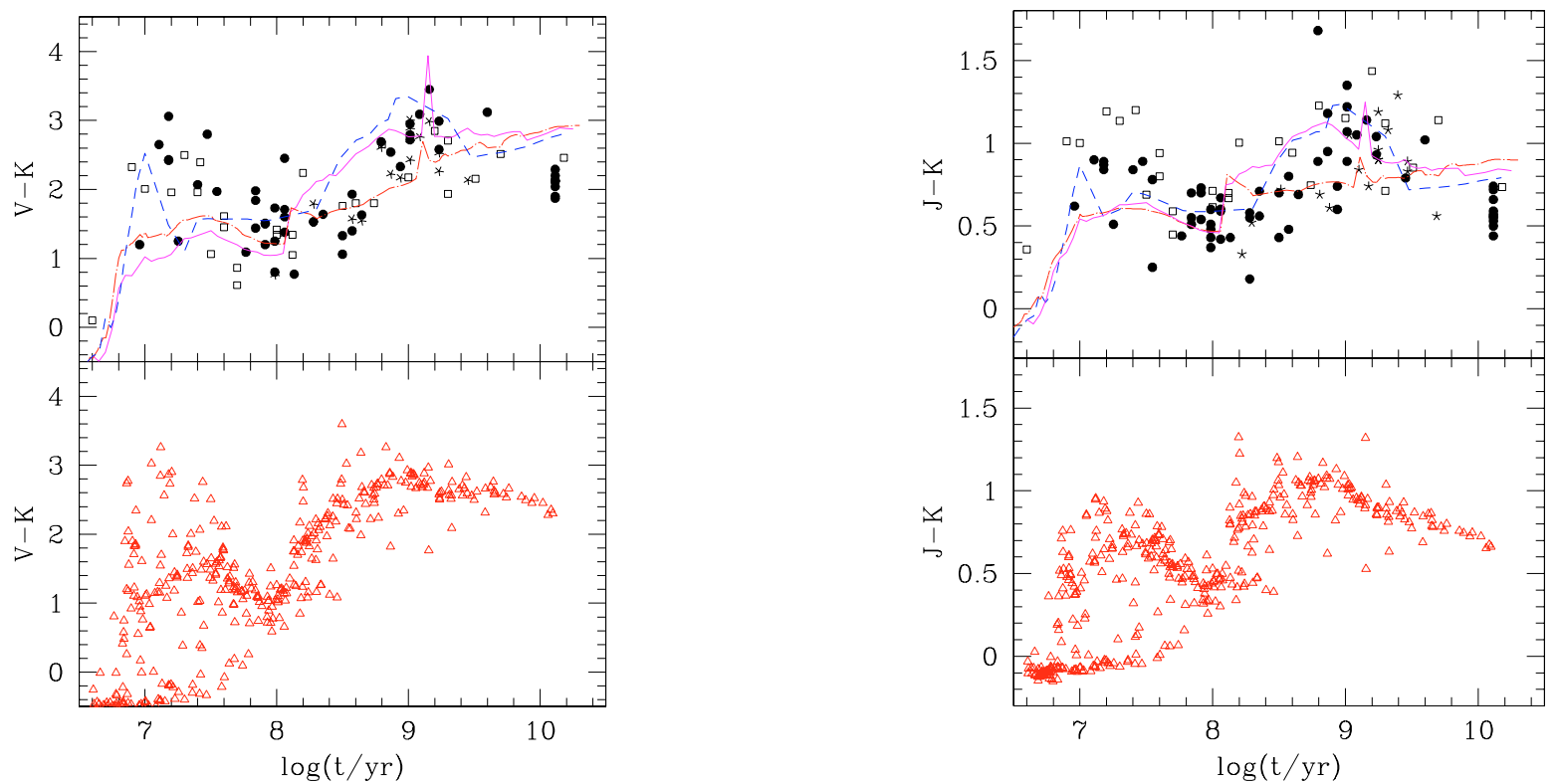

Fig. 9. Broad-band $V-K$ and $J-K$ colours of LMC clusters as a function of cluster age. Top panels: observed photometric data taken from various compilations: Persson et al. (1983, filled circles); Kyeong et al. (2003, empty squares); and the $V$-band photometry by Goudfrooij et al. (2006) adopting an aperture radius of $50 \mathrm{arcsec}$, combined to the $J H K_{\mathrm{s}}$ photometry from Pessev et al. (2006; starred symbols). Clusters are assigned ages by adopting the $S$-parameter-age calibration by Girardi et al. (1995). Predicted SSP colours from various authors are superimposed for comparison: this work (solid line, $Z=0.008$ ), Maraston (2005; dashed line, $Z=Z_{\odot} / 2$ ), Bruzual \& Charlot (2003; dot-dashed line, $Z=0.008$ ). We note that the spike in the colour evolution at $\sim 10^{9} \mathrm{yr}$ is real and is explained in detail by Girardi \& Bertelli (1998). Bottom panels: simulations of stellar clusters based on the new isochrones. They roughly follow the distribution of typical sizes (or initial total masses) and metallicities of LMC clusters. The age distribution is assumed to be constant. The simulated sample has a limiting magnitude of $M_{V}^{\lim }=-6.5$, which roughly corresponds to the one of the observed samples in the top panel.

single-burst stellar populations (SSP) models (solid line) as due to the development of the TP-AGB phase. We refer the reader to Appendix A for a discussion on the behaviour of $V-K$ at ages $t \sim 10^{8} \mathrm{yr}$ and its relation to the evolution of the most massive AGB stars. Integrated magnitudes are calculated accounting for the luminosity variations (i.e. the long-lived luminosity dips in low-mass stars) driven by thermal pulses, and the effect on the emitted spectrum by circumstellar dust.

For comparison we also show the results of other two widelyused SSP models, namely Charlot \& Bruzual (2003; dot-dashed line) and Maraston (2005; dashed line). A few points should be noticed in respect to the age interval $t \gtrsim 10^{8} \mathrm{yr}$, when AGB stars are present. While Charlot \& Bruzual (2003) colours tend to be bluer than the observations because of their likely underestimation of the TP-AGB phase (see Bruzual 2007, for a recent revision of this aspect in their models), Maraston (2005) tends to form an upper envelope to the data, especially in $V-K$. Our results are intermediate between the two.

The bottom panels of Fig. 9 illustrate the same colour evolution, by means of simulated clusters. The cluster simulations are performed by randomly adding stars to the isochrones, with the probability of a given initial stellar mass given by the Chabrier (2001) log-normal initial mass function. The addition of stars to a simulated cluster is stopped when its initial total mass reaches a given value $M_{\mathrm{T}}$. The entire cluster sample is assumed to follow a $M_{\mathrm{T}}^{-1.5}$ distribution, which is derived from the luminosity function of LMC clusters (Elson \& Fall 1985; see also Girardi et al. 1995, Sect. 8.2). The cluster metallicities follow the LMC agemetallicity relation from Pagel \& Tautvaišienè's (1998) bursting model, which has been shown to reproduce the observed trend. The distribution of cluster ages is assumed to be constant; although this is not a realistic assumption (see Girardi et al. 1995), it allows us a good illustration of the colour evolution over the complete age range. A posteriori, we impose a limiting magnitude of $M_{V}^{\mathrm{lim}}=-6.5 \mathrm{mag}$ to the simulated sample, which roughly corresponds to that of the observed samples in the top panels of Fig. 9. The results present the intrinsic colour dispersion expected for the cluster data due to their small numbers of evolved stars (stochastic fluctuations), and in this aspect they look clearly more similar to the observations than the continuous narrow SSP lines depicted in the top panel. The main discrepancy between models and simulations is at ages younger than $10^{8} \mathrm{yr}$, where a bunch of very blue clusters is predicted, but absent from the observed sample ${ }^{12}$. The blue clusters are essentially those for which no red super-giant was predicted, and derive naturally from the fact that the red super-giant phase is short-lived. In order to recover the observed colours of the youngest clusters, the red super-giant phase has to be significantly longer and cooler than in the Bertelli et al. (1994) isochrones. On one hand, a longer duration could be attained, for instance, by invoking a larger efficiency of overshoot, but this may in turn inhibit the development of the blue loops of massive stars (see the discussion in Alongi et al. 1991). On the other hand, the effective temperatures of red super-giants depend very much on the treatment of the density inversion that is predicted to take place in the outermost atmospheric layers. A deeper analysis of this issue is beyond the scope of the paper and it is postponed to future work.

Another questionable point involves the ages between $10^{8} \mathrm{yr}$ and $\sim 4 \times 10^{8} \mathrm{yr}$, after the AGB phase develops: in this age interval, our models become on the mean redder than the data. As mentioned in the Appendix, removing this feature from the models poses a theoretical difficulty as it would as well prevent the significant enrichment of helium and nitrogen in the most massive AGB stars, which is instead required to explain the observed

12 Isochrones in this age range were taken from Bertelli et al. (1994). 
abundances in planetary nebulae of type I. Finally, at older ages the adoption of an age-metallicity relation allows us to reproduce the colours of the oldest LMC clusters, which have ages and metallicities typical of old Galactic globular clusters.

In short, these isochrones offer a valid alternative for the modelling of the integrated light of star clusters and galaxies, since they pass the LMC cluster test performing similarly well as the widely-used Charlot \& Bruzual (2003) and Maraston (2005) SSP models, when we look at the mean colour evolution (top panels of Fig. 9). Moreover, the isochrones we provide allow the simulation of resolved stellar populations, which is essential for their further testing - as illustrated by the simulations in the bottom panels of Fig. 9. The isochrones are based on internally consistent sets of tracks, which naturally obey the fuel consumption theorem, and provide other quantities necessary for a consistent modelling of galaxies - namely the chemical yields and remnant masses (see Marigo \& Girardi 2001, for a discussion on this latter point). Having all these components together allows us the simultaneous comparison of the model results with many different observables, indicating hidden problems (like the young blue clusters predicted in Fig. 9) and hopefully pointing the way to future improvements in the models.

\section{Data tables and final remarks}

\subsection{Data tables}

The data presented in this paper are provided in two different ways:

(1) as a repository of static tables down-loadable from the VizieR Catalogue Service at CDS (http://vizier. u-strasbg.fr) and from the URL http://stev.oapd. inaf.it/dustyAGB07. This database includes not only the isochrones, but also bolometric corrections, extinction coefficients, dust attenuation curves, etc.

(2) via an interactive web interface at http://stev.oapd. inaf.it/cmd, that allows users to build isochrones (or sequences of them) for different values of age and metallicity, photometric systems, and dust properties. The same web form provides access to a series of other services, like the computation of luminosity functions, integrated magnitudes, and simulated star clusters.

All information about these data are provided in the form of either ReadMe files in case (1), or HTML help pages in case (2). Most of the information available will be of interest only to the people who actually access the data, and therefore will not be repeated here. Suffice it to recall that isochrones are provided for any metal content in the range $0.0001 \leq Z \leq 0.03$ (corresponding to populations with $-2.31 \leq[\mathrm{M} / \mathrm{H}] \leq+0.22$, for scaled-solar initial compositions), and ages in the range $7.8 \leq$ $\log (t / \mathrm{yr}) \leq 10.2$ (i.e., from 0.063 to $15.8 \mathrm{Gyr}$ ). The initial mass ranges from 0.15 to $7 M_{\odot}$.

Moreover, the http://stev. oapd.inaf.it/cmd URL allows the mass range to be extended from 7 to $100 M_{\odot}$, using the tracks from Bertelli et al. (1994) isochrones. This allows the age range to be extended down to about $4 \mathrm{Myr}$ for complete isochrones - or even down to $t=0$ in case one is interested in obtaining an extended zero-age main sequence. The only problem is that the tracks for massive stars are not available for $Z_{\odot} \leq Z \leq 0.03$, and in such a case have to be taken from the $Z=0.02$ set of Bertelli et al. (1994).

The basic information provided along isochrones consists of the luminosity, effective temperature, surface gravity, and absolute magnitudes in the photometric system under consideration. For the TP-AGB phase, this information is complemented with the core mass, surface $\mathrm{C} / \mathrm{O}$ ratio, pulsation mode and period, and mass-loss rate. The TP-AGB section of an isochrone can be easily identified by the information about the core mass, whereas the $\mathrm{C}$-star phase is identified by $\mathrm{C} / \mathrm{O}>1$. These latter variables are also useful to simulate, a posteriori, the luminosity and $T_{\text {eff }}$ variations caused by pulse cycles on the TP-AGB, as we have done in a series of papers (e.g., Marigo et al. 2003; Cioni et al. 2006; Girardi \& Marigo 2007b).

Finally, we remark that the http://stev. oapd.inaf.it/ cmd web interface is going to be continuously expanded, either with new extensions to the tracks (e.g., with the planetary nebulae and white dwarf sequences), new photometric systems, new spectra from which to derive bolometric corrections, and different dust compositions. Interstellar extinction will be included in the isochrones in a consistent way (Girardi et al. 2007, in prep.). In any case, the original database, as here described, will remain available as well.

\subsection{Final remarks}

Users of the new isochrones should be aware that:

- The isochrones are expected to perform reasonably well for AGB populations in the Milky Way disc and in the Magellanic Clouds; they have already been compared or calibrated against the latter data. The quality of the results at very low metallicities $([\mathrm{Fe} / \mathrm{H}] \lesssim-1.5)$ is yet to be verified.

- Present-day model atmospheres for cool stars are still far from satisfactory. Molecular line lists are acknowledged to be incomplete, whereas for LPVs dynamical model atmospheres would probably provide more realistic spectral energy distributions than the static models we have adopted for both M- and C-type stars. The template spectra used in our models do not sufficiently cover the expected range of metallicities, effective temperatures, and surface gravities of AGB populations in resolved galaxies.

- Dust properties may differ from the compositions assumed in this work, and they likely change along the TP-AGB evolution as a function of parameters other than those considered here. For a given dust composition, RT models have been calculated for a large range of optical depths, even if particular combinations of dust and mass-loss rate are unlikely. For example, pure $\mathrm{AlOx}$ is preferentially seen in stars with low mass-loss rates only, and the combination of AlOx and silicates is most appropriate for intermediate mass-loss rates. For the Galaxy, the mass-loss rate below which the pure AlOx models should probably be used, and above which pure silicates are likely to be most appropriate are, respectively, about $10^{-7} M_{\odot} \mathrm{yr}^{-1}$ and $10^{-6} M_{\odot} \mathrm{yr}^{-1}$. At different metallicities these ranges may be different and, therefore, we calculated the full range of models for all compositions considered here. An extended list of other caveats associated with dusty circumstellar envelopes is presented in G06.

- Mass-loss prescriptions from evolved stars are still very uncertain, and probably none of the presently available formalisms can be applied with confidence. For O-rich AGB stars, even the basic physical process driving mass loss is being debated (see Woitke 2006).

- In constructing our theoretical isochrones, we have made all possible effort to ensure internal consistency, but this has not always been possible. For instance, the dust condensation prescriptions adopted here are likely inconsistent with those 
assumed in the hydrodynamic calculations from which the adopted mass-loss formulas were derived. On the other hand, the uncertainties resulting from such inconsistencies are no more important than those resulting from a fundamental lack of understanding of certain aspects of stellar evolution and mass loss.

Work is in progress to verify or improve on the points above; in particular, we are performing an extensive study of the Magellanic Cloud data (from $I$ to [24], in preparation), and checking the results at low metallicities with data for dwarf galaxies and globular clusters (Gullieuszik et al. 2007a,b, and work in preparation). Model atmospheres of cool giants are being extended by Aringer et al. (in prep.).

Despite the caveats, we believe that our new isochrones constitute significant progress. We recall that the simple discussion of the above mentioned points would have been meaningless in the context of the more crude TP-AGB isochrones listed in Table 1.

In fact, we believe this is the first extended set of isochrones that includes the TP-AGB evolutionary phase with the level of completeness and detail it deserves. Crucial effects such as the third dredge-up (which makes C-type stars), hot bottom burning (which largely prevents the most massive AGB stars from becoming C-type), and variable molecular opacities (which causes the cool red tail of $\mathrm{C}$ stars in near- and mid-IR CMDs), have been considered. Mass-loss rates are described with a formalism that discriminates between C- and M-type stars, and leads to the attainment of the super-wind regime during the latest stages of the TP-AGB evolution. The transition from the first overtone to the fundamental mode is predicted, which helps trigger higher mass-loss rates. Parameters associated with the third dredge-up are calibrated so as to reproduce the $\mathrm{C}$-star luminosity functions observed in the Magellanic Clouds, and the lifetimes of $\mathrm{C}$ - and M-type stars derived from star counts in Magellanic Cloud clusters. Many photometric systems are considered, properly taking into account the different spectra of O-rich and C-rich stars, including the stellar obscuration by circumstellar dust at optical to near-IR wavelengths, and its dust emission in the mid- and farIR. We vary the expansion velocity of the wind and the dust-togas ratio consistently as a function of stellar parameters $\left(\dot{M}, T_{\text {eff }}\right.$, $L, \mathrm{C} / \mathrm{O}, Z$ ), in place of simpler parametrizations often used in the literature. Dust reprocessing of radiation makes the most evolved AGB stars disappear in optical pass-bands, and appear at a very extended red tail in near- and mid-IR CMDs. All these features make the new isochrones potentially very useful in the study of systems containing AGB stars, both resolved and unresolved.

The new models have been included in the TRILEGAL code (Girardi et al. 2005; Girardi \& Marigo 2007b) ${ }^{13}$ for the simulation of resolved stellar populations in the Milky Way and in external galaxies, and in the GRASIL code (GRAphite and SILicate; Silva et al. 1998; Panuzzo et al. 2005) ${ }^{14}$ for computing the integrated spectrum of galaxies from the X-ray to the radio domain taking into account the effects of dust. Forthcoming papers will present applications of both codes, including the extensive testing of our TP-AGB tracks and dust models against observational data.

Acknowledgements. This study was supported by the University of Padova (Progetto di Ricerca di Ateneo CPDA052212), COFIN INAF 2005, and

\footnotetext{
13 http://trilegal.ster. kuleuven.be/ or http: //stev.oapd.inaf.it/trilegal

14 http://adlibitum.oat.ts.astro.it/silva/default.html or http://web.oapd.inaf.it/granato/grasil/grasil.html
}

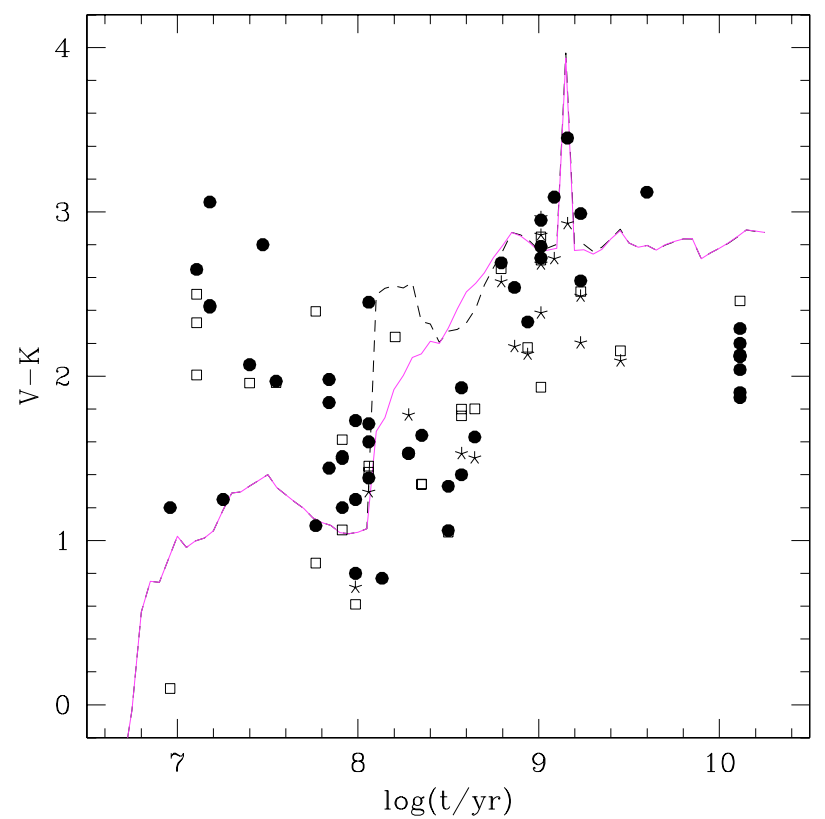

Fig. A.1. Integrated $V-K$ colours as a function of SSP age, derived from the original Paper I TP-AGB tracks (dashed line), and after the revision applied to the TP-AGB models with initial masses $\geq 3.0 M_{\odot}$ (solid line). The adopted metallicity is $Z=0.008$. Observed colours of LMC clusters are taken from the compilations by Persson et al. (1983; filled circles); Kyeong et al. (2003, empty squares); and Pessev et al. (2006, starred symbols).

contract ASI-INAF I/016/07/0. We acknowledge Rita Gautschy-Loidl for providing us the C-star spectra, and Bernhard Aringer for the help in implementing them. We thank Anil Seth, Ben Williams, Enrico Held, Marco Gullieuszik, Leandro Kerber, and Mauro Barbieri for pointing out some bugs and problems in preliminary versions of these isochrones, and Jacco van Loon for his very useful suggestions.

\section{Appendix A: Revised evolution of massive TP-AGB models}

The TP-AGB evolution of more massive stars, with initial masses $3.0 \leq M / M_{\odot} \leq 5.0$, has been modified as follows. The starting point that has stimulated the revision is the behaviour of the integrated colours (e.g. $V-K, J-K, H-K$ ) as a function of age as displayed by the Magellanic Clouds' clusters of age $t \gtrsim 10^{8}$ yr. As shown in Fig. A.1, the comparison with SSP colours, derived from the isochrones including the original TP-AGB tracks from Paper I, would indicate a sizable excess in the predicted $V-K$ colours at ages around $1-4 \times 10^{8} \mathrm{yr}$. The sudden jump to $V-K \sim 2.5$ at $t \sim 10^{8} \mathrm{yr}$ is due to the occurrence of the AGB phase in models with $M_{\mathrm{i}} \sim 5 M_{\odot}$, which corresponds to the maximum initial mass (for our set of models) required to develop an electron-degenerate $\mathrm{C}-\mathrm{O}$ core. In the $1-4 \times 10^{8}$ yr age range, while the integrated $V$-band luminosity is essentially produced by less-evolved stars still populating the region close to the main-sequence turn-off (see e.g., Charlot \& Bruzual 1991), a significant contribution in the $K$-band comes from the most massive AGB stars, with initial masses in the range $3.0 M_{\odot} \leq M_{\mathrm{i}} \leq 5.0 M_{\odot}$.

It follows that the $V-K$ colour jump at $t \sim 10^{8} \mathrm{yr}$ should be ascribed to an overestimation of the TP-AGB phase of more massive AGB models, which translates into either a) too high luminosities and/or b) too long lifetimes. Of course these aspects 
are interrelated as they are both highly sensitive to model details dealing with two crucial processes that affect the evolution of these stars, namely HBB and mass loss by stellar winds.

Given the high degree of uncertainty that affects the theoretical predictions for $\dot{M}$ along the AGB phase, the theoretical discrepancy is likely attributed to an underestimation of the massloss efficiency in massive AGB stars. A simple remedy could be obtained artificially just introducing a multiplicative factor in front of our mass-loss law (similar to the $\eta$ parameter of the Reimers law) for $M_{\mathrm{i}} \gtrsim 4.0 M_{\odot}$, but in this case we would introduce a discontinuity in our adopted formalism for $\dot{M}$, as the correction factor should then vanish at lower $M_{\mathrm{i}}$.

After a general re-consideration of the many prescriptions of our synthetic TP-AGB model, we have found that a more physically-sound way to obtain higher mass-loss rates may come from an improved treatment of the pulsation properties of the most massive AGB stars, compared to the original description given in Paper I.

Specifically, following the results of non-adiabatic pulsation models of Fox \& Wood (1982) for stellar masses 3-6 $M_{\odot}$, it seems likely that in Paper I the switching of the pulsation mode from the first overtone to the fundamental one has been assumed to occur at too high luminosities in the most massive TP-AGB models.

We recall that the critical luminosity, $L_{1-0}$, is defined as the luminosity at which the growth rates of the first overtone mode and the fundamental mode are equal. In Paper I the critical luminosity has been derived from the pulsation models calculated by Ostlie \& Cox (1986) in the mass range $0.8-2.0 M_{\odot}$, and the fitting relation is given by

$$
\begin{aligned}
\log \left(L_{1-0} / L_{\odot}\right)_{\mathrm{MG} 07}= & -14.516+2.277 \log \left(M / M_{\odot}\right) \\
& +5.046 \log T_{\text {eff }}-0.084 \log (Z / 0.02) .
\end{aligned}
$$

In Fig. A.2, we compare $L_{1-0}$ as predicted by the original Paper I prescription (solid line), extended over the entire mass range of interest, with the results from Fox \& Wood (1982). We see that while at $M=3 M_{\odot}$ Eq. (A.2) is fully consistent with the results from Fox \& Wood (1982), at $M=6 M_{\odot},\left(L_{1-0} / L_{\odot}\right)_{\mathrm{MG} 07}$ exceeds significantly the range of critical luminosities derived from the Fox \& Wood (1982) data. Based on these results we apply a rough correction $\Delta \log L_{\text {cor }}$ to Eq. (A.2) to decrease the transition luminosity for $M \gtrsim 3.0 M_{\odot}$, so that we write

$$
\log \left(L_{1-0} / L_{\odot}\right)=\log \left(L_{1-0} / L_{\odot}\right)_{\mathrm{MG} 07}+\Delta \log L_{\text {cor }}
$$

with

$\Delta \log L_{\mathrm{cor}}=-0.125\left(M / M_{\odot}-2.5\right)$.

The revised relation for $L_{1-0} / L_{\odot}$ is also plotted in Fig. A.2 (dashed line). We see that it coincides with the old relation for $M \leq 2.5 M_{\odot}$, while it flattens to lower luminosities for larger masses. It should be observed that, given the paucity of the theoretical pulsation models presently available, this revision is meant to be just a crude attempt to improve our treatment of $L_{1-0}$. Indeed, new pulsation models over wider ranges of the key parameters (i.e. mass, metallicity, $\mathrm{C} / \mathrm{O}$ ratio, etc.) are urgently required to allow for a real progress in modelling the pulsation properties of AGB stars.

The new formula for the critical luminosity $L_{1-0}$ is used to re-calculate the TP-AGB evolution of models with initial masses $3.0 \leq M / M_{\odot} \leq 5.0$ for all choices of the metallicity. Massive models start to pulsate in the fundamental mode at lower luminosities than assumed in Paper I, which favours the attainment of larger mass-loss rates with consequent earlier termination of

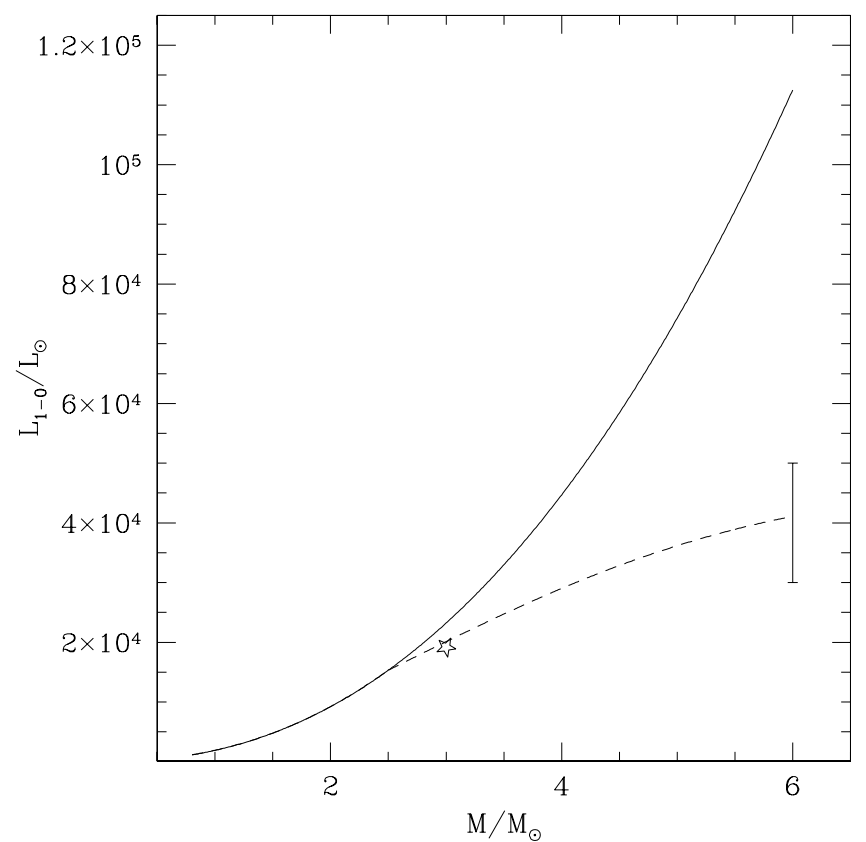

Fig. A.2. Transition luminosity defining the switching from fundamental mode to first-overtone pulsation mode, as a function of the current stellar mass, assuming $Z=0.008$ and $\log T_{\text {eff }}=3.52$. The original relation of Paper I is shown as a solid line (Eq. (A.2)), while the modified relation at higher stellar masses (Eq. (A.2)) is drawn with a dashed line. The starred symbol corresponds to a pulsation model by Fox \& Wood (1982) with $M_{\mathrm{i}}=3.0 M_{\odot}, Z=0.019, \log \left(T_{\text {eff }}\right)=3.49$ ), while the vertical bar roughly defines the range of critical luminosity for a few models calculated by Fox \& Wood (1982) with $M_{\mathrm{i}}=6 M_{\odot}$.

the AGB phase. This effect is more pronounced at larger masses $\left(M \geq 4 M_{\odot}\right.$ ) shortening the TP-AGB lifetimes by a factor of $2-5$. The impact on the integrated $V-K$ colours is good as we see in Fig. A.1. The jump at $t \sim 10^{8} \mathrm{yr}$ is reduced and a more gradual increase of the colour proceeds at larger ages, allowing for the seeked consistency with the observations. Similar results are obtained for other integrated colours, like $J-K$, and $H-K$.

It should also be emphasized that the TP-AGB fuel at ages $t \gtrsim 10^{8} \mathrm{yr}$ cannot be arbitrarily cut down since otherwise we would run into other problems related to the observed properties of the most luminous AGB stars and their progeny. For instance, we have verified that even invoking an extreme shortening of the TP-AGB phase for these stars, the predicted integrated $V-K$ and $J-K$ colours are still consistent with the observed ones, but in this case we cannot fulfill the nucleosynthesis constraint set by type I planetary nebulae. Such objects are characterized by high $\mathrm{He}$ and $\mathrm{N}$ abundances, which are currently interpreted as the result of hot-bottom burning in more massive AGB stars (e.g., Marigo et al. 2003b; Pottasch \& Bernard-Salas 2006). An overly-short activation of the CNO cycle at the base of the convective envelope would not allow the massive AGB progenitors to reach the He and $\mathrm{N}$ surface enrichment measured in type I planetary nebulae. In other words, the nuclear fuel burnt during the TP-AGB phase cannot be constrained only on the basis of the integrated luminosities, but it should also be checked against indications provided by abundance analyses in the stellar ejecta. In fact, as extensively discussed by Marigo \& Girardi (2001) a precise fraction of the nuclear fuel consumption (emitted light) in post-main sequence stars ends in the form of chemical (mainly $\mathrm{He}, \mathrm{N}, \mathrm{O}$ ) yields. One cannot produce large amounts of $\mathrm{He}$ and 
$\mathrm{N}$ in the most massive AGB stars (as observed in their PN descendants), without adding a marked contribution of these same stars to the integrated light.

\section{References}

Alexander, D. R., \& Ferguson, J. W. 1994, ApJ, 437, 879

Allard, F., Hauschildt, P. H., Alexander, D. R., Tamanai, A., \& Ferguson, J. W. 2000, in From giant planets to cool stars, ed. C. A. Griffith, \& M. S. Marley, ASP Conf. Ser., 212, 127

Alongi, M., Bertelli, G., Bressan, A., \& Chiosi, C. 1991, A\&A, 244, 95

Bahcall, J. N., Pinsonneault, M. H., \& Wasserburg, G. J. 1995, Rev. Mod. Phys., $67,4,781$

Begemann, B., Dorschner, J., Henning, Th., et al. 1997, ApJ, 476, 199

Bergeat, J., Knapik, A., \& Rutily, B. 2001, A\&A, 369, 178

Bertelli, G., Bressan, A., Chiosi, C., Fagotto, F., \& Nasi, E. 1994, A\&AS, 106, 275

Bessell, M. S. 1990, PASP, 102, 1181

Bessell, M. S., \& Brett, J. M. 1988, PASP, 100, 1134

Bessell, M. S., Castelli, F., \& Plez, B. 1998, A\&A, 333, 231

Blommaert, J. A. D. L., Groenewegen, M. A. T., Okumura, K., et al. 2006, A\&A, 460, 555

Blum, R. D., Mould, J. R., Olsen, K. A., et al. 2006, AJ, 132, 2034

Bohlin, R. C. 2007, in The Future of Photometric, Spectrophotometric and Polarimetric Standardization, ed. C. Sterken, ASP Conf. Ser., 364, 315

Bolatto, A. D., Simon, J. D., Stanimirović, S., et al. 2007, ApJ, 655, 212

Bonatto, C., Bica, E., \& Girardi, L. 2004, A\&A, 415, 571

Boothroyd, A. I., \& Sackmann, I.-J. 1988, ApJ, 328, 641

Bowen, G. H. 1988, ApJ, 329, 299

Bowen, G. H., \& Willson, L. A. 1991, ApJ, 375, L53

Bressan, A., Granato, G. L., \& Silva, L. 1998, A\&A, 332, 135 (B98)

Bruzual, A. G. 2007, in Stellar populations as building blocks of galaxies, ed. A.

Vazdekis, \& R. Peletier (Cambridge University Press), IAU Symp., 241, in press [arXiv: astro-ph/0703052]

Calura, F., Pipino, A., \& Matteucci, F. 2007, A\&A, 479, 669

Cardelli, J. A., Clayton, G. C., \& Mathis, J. S. 1989, ApJ, 345, 245

Castelli, F., \& Kurucz, R. L. 2003, in Modelling of Stellar Atmospheres, ed. N.

Piskunov, W. W. Weiss, \& D. F. Gray (ASP), IAU Symp., 210, A20

Castelli, F., Gratton, R. G., \& Kurucz, R. L. 1997, A\&A, 318, 841

Catelan, M. 2000, ApJ, 531, 826

Catelan, M. 2007 [arXiv:0708.2445]

Chabrier, G. 2001, ApJ, 554, 1274

Chabrier, G., Baraffe, I., Allard, F., \& Hauschildt, P. H. 2000, ApJ, 542, 464

Charlot, S., \& Bruzual, G. 1991, ApJ, 367, 126

Charlot, S., \& Bruzual, G. 2003, MNRAS, 344, 1000

Cioni, M.-R., Habing, H. J., Loup, C., et al. 1999, in The stellar content of Local Group galaxies, ed. P. Whitelock, \& R. Cannon (ASP), IAU Symp., 192, 65

Cioni, M.-R.L., Girardi, L., Marigo, P., \& Habing, H. J. 2006, A\&A, 448, 77

Cohen, M., Wheaton, W. A., \& Megeath, S. T. 2003, AJ, 126, 1090

Cordier, D., Pietrinferni, A., Cassisi, S., \& Salaris, M. 2007, AJ, 133, 468

David, P., \& Pégourié, B. 1995, A\&A, 293, 833

Decin, L., \& Eriksson, K. 2007, A\&A, 472, 1041

Dijkstra, C., Speck, A. K., Reid, R. B., \& Abraham, P. 2005, ApJ, 633, L133

Dwek, E. 1998, ApJ, 501, 643

Elitzur, M., \& Ivezić, Ž. 2001, MNRAS, 327, 403

Elson, R. A. W., \& Fall, S. M. 1985, PASP, 97, 692

Ferrarotti, A. S. 2003, Ph.D. Thesis, Universität Heidelberg

Ferrarotti, A. S., \& Gail, H.-P. 2001, A\&A, 371, 133

Ferrarotti, A. S., \& Gail, H.-P. 2002, A\&A, 382, 256

Ferrarotti, A. S., \& Gail, H.-P. 2006, A\&A, 557, 553

Finley, D. S., Koester, D., \& Basri, G. 1997, ApJ, 488, 375

Fluks, M. A., Plez, B., Thé, P. S., et al. 1994, A\&AS, 105, 311

Fouqué, P., Chevallier, L., Cohen, M., et al. 2000, A\&AS, 141, 313

Fox, M. W., \& Wood, P. R. 1982, ApJ, 259, 198

Frost, C. A., Cannon, R. C., Lattanzio, J. C., Wood, P. R., \& Forestini, M. 1998, A\&A, 332, L17

Frogel, J. A., Mould, J., \& Blanco, V. M. 1990, ApJ, 352, 96

Fukugita, M., Ichikawa, T., Gunn, J. E., et al. 1996, AJ, 111, 1748

Geisler, D. 1996, AJ, 111, 480

Girardi, L. 1999, MNRAS, 308, 818

Girardi, L., \& Bica, E. 1993, A\&A, 274, 279

Girardi, L., \& Bertelli, G. 1998, MNRAS, 300, 533

Girardi, L., \& Marigo, P. 2007a, A\&A, 462, 237
Girardi, L., \& Marigo, P. 2007b, in Why galaxies care about AGB stars: their importance as actors and probes, ed. Kerschbaum, C. Charbonnel, \& R. F. Wing, ASP Conf. Ser., 378, 20

Girardi, L., Chiosi, C., Bertelli, G., \& Bressan, A. 1995, A\&A, 298, 87

Girardi, L., Bressan, A., Bertelli, G., \& Chiosi, C. 2000, A\&AS, 141, 371 (Gi00) Girardi, L., Bertelli, G., Bressan, A., et al. 2002, A\&A, 391, 195

Girardi, L., Grebel, E. K., Odenkirchen, M., \& Chiosi, C. 2004, A\&A, 422, 205

Girardi, L., Groenewegen, M. A. T., Hatziminaoglou, E., \& da Costa, L. 2005, A\&A, 436, 895

Goudfrooij, P., Gilmore, D., Kissler-Patig, M., \& Maraston, C. 2006, MNRAS, 369,697

Granato, G. L., \& Danese, L. 1994, MNRAS, 268, 235

Grebel, E. K., \& Roberts, W. J. 1995, A\&AS, 109, 293

Grevesse, N., \& Noels, A. 1993, Phys. Scr. T, 47, 133

Groenewegen, M. A. T. 1993, Ph.D. Thesis, University of Amsterdam (available at http://www.ster.kuleuven.be/ groen/thesis.ps.gz)

Groenewegen, M. A. T. 1995, A\&A, 293, 463

Groenewegen, M. A. T. 2004, A\&A, 425, 595

Groenewegen, M. A. T. 2006, A\&A, 448, 181 (G06)

Groenewegen, M. A. T., \& de Jong, T. 1993, A\&A, 267, 410

Groenewegen, M. A. T., \& de Jong, T. 1994a, A\&A, 282, 127

Groenewegen, M. A. T., \& de Jong, T. 1994b, A\&A, 283, 463

Groenewegen, M. A. T., \& de Jong, T. 1994c, A\&A, 288, 782

Groenewegen, M. A. T., Whitelock, P. A., Smith, C. H., \& Kerschbaum, F. 1998, MNRAS, 293, 18

Groenewegen, M. A. T., Sevenster, M., Spoon, H. W. W., \& Perez, I. 2002, A\&A, 390,511

Hauck, B., \& Mermilliod, M. 1998, A\&AS, 129, 431

Herwig, F. 2000, A\&A, 360, 952

Hewett, P. C., Warren, S. J., Leggett, S. K., \& Hodgkin, S. T. 2006, MNRAS, 367,454

Höfner, S., Sandin, C., Aringer, B., et al. 2003, in Modelling of Stellar Atmospheres, ed. N. Piskunov, W. W. Weiss, \& D. F. Gray (ASP), IAU Symp., 210,353

Holtzman, J. A., Burrows, C. J., Casertano, S., et al. 1995, PASP, 107, 1065

Homeier, D., Koester, D., Hagen, H.-J., et al. 1998, A\&A, 338, 563

Ireland, M. J., \& Scholz, M. 2006, MNRAS, 367, 1585

Ivezić, Ž., Nenkova, M., \& Elitzur, M. 1999, User Manual for DUSTY, University of Kentucky Internal Report, accessible at http://www . pa.uky. edu/ moshe/dusty

Izzard, R. G., Tout, C. A., Karakas, A. I., \& Pols, O. R. 2004, MNRAS, 350, 407

Kyeong, J.-M., Tseng, M.-J., \& Byun, Y.-I. 2003, A\&A, 409, 479

Lagadec, E., Zijlstra, A. A., Sloan, G. C., et al. 2007, MNRAS, 376, 1270

Laor, A., \& Draine, B. T. 1993, ApJ, 402, 441

Lebzelter, Th., Posch, Th., Hinkle, K., Wood, P. R., \& Bouwman, J. 2006, ApJ, 635, L145

Loidl, R., Lançon, A., \& Jørgensen, U. G. 2001, A\&A, 371, 1065

Lupton, R. H., Gunn, J. E., \& Szalay, A. S. 1999, AJ, 118, 1406

Maíz Apellániz, J. 2006, AJ, 131, 1184

Maíz-Apellániz, J. 2007, in The Future of Photometric, Spectrophotometric and Polarimetric Standardization, ed. C. Sterken (ASP), ASP Conf. Ser., 364, 227 Maraston, C. 2005, MNRAS, 362, 799

Marigo, P. 2002, A\&A, 387, 507

Marigo, P. 2007, in Why galaxies care about AGB stars: their importance as actors and probes, ed. Kerschbaum, C. Charbonnel, \& R. F. Wing, ASP Conf. Ser., 378, 392

Marigo, P., \& Girardi, L. 2001, A\&A, 377, 132

Marigo, P., \& Girardi, L. 2007, A\&A, 469, 239 (Paper I)

Marigo, P., Girardi, L., \& Bressan, A. 1999, A\&A, 344, 123

Marigo, P., Girardi, L., \& Chiosi, C. 2003a, A\&A, 403, 225

Marigo, P., Bernard-Salas, J., Pottasch, S. R., Tielens, A. G. G. M., \& Wesselius, P. R. 2003b, A\&A, 409, 619

Marshall, J. R., van Loon, J. Th., Matsuura, M., et al. 2004, MNRAS, 355, 1348 Matsuura, M., Zijlstra, A. A., Bernard-Salas, J., et al. 2007, MNRAS, 382, 1889 Morgan, H. L., \& Edmunds, M. G. 2003, MNRAS, 343, 427

Moro, D., \& Munari, U. 2000, A\&AS, 147, 361

Mouhcine, M. 2002, A\&A, 394, 125

Nikolaev, S., \& Weinberg, M. D. 2000, ApJ, 542, 804

Ostlie, D. A., \& Cox, A. N. 1986, ApJ, 311, 864

Pagel, B. E. J., \& Tautvaišienè, G. 1998, MNRAS, 299, 535

Panuzzo, P., Silva, L., Granato, G. L., Bressan, A., \& Vega, O. 2005, in The Spectral Energy Distributions of Gas-Rich Galaxies: Confronting Models with Data, ed. C. C. Popescu, \& R. J. Tuffs., AIP Conf. Proc., 761, 187 Pégourié, B. 1988, A\&A, 194, 335

Pessev, P. M., Goudfrooij, P., Puzia, T. H., \& Chandar, R. 2006, AJ, 132, 781

Persson, S. E., Aaronson, M., Cohen, J. G., Frogel, J. A., \& Matthews, K. 1983, ApJ, 266, 105

Piovan, L., Tantalo, R., \& Chiosi, C. 2003, A\&A, 408, 559 
Pottasch, S. R., \& Bernard-Salas, J. 2006, 457, 189

Raimondo, G., Brocato, E., Cantiello, M., \& Capaccioli, M. 2005, AJ, 130, 2625

Reach, W. T., Megeath, S. T., Cohen, M., et al. 2005, PASP, 117, 978

Reimers, D. 1975, Société Royale des Sciences de Liège, Mémoires, 8, 369

Renzini, A., \& Fusi Pecci, F. 1988, ARA\&A, 26, 199

Rouleau, F., \& Martin, P. G. 1991, ApJ, 377, 526

Rowan-Robinson, M., Lock, T. D., Walker, D. W., \& Harris, S. 1986, MNRAS, 222,273

Schröder, K.-P., Wachter, A., \& Winters, J. M. 2003, A\&A, 398, 229

Silva, L., Granato, G. L., Bressan, A., \& Danese, L. 1998, ApJ, 509, 103

Sirianni, M., Jee, M. J., Benítez, N., et al. 2005, PASP, 117, 1049

Sloan, G. C., Kraemer, K. E., Matsuura, M., et al. 2006, ApJ, 645, 1118

Speck, A. K., Cami, J., Markwick-Kemper, C., et al. 2006, ApJ, 650, 892

Todini, P., \& Ferrara, A. 2001, MNRAS, 325, 726

Thompson, G. D., Corman, A. B., Speck, A. K., \& Dijkstra, C. 2006, ApJ, 652, 1654

van der Bliek, N. S., Gustafsson, B., \& Eriksson, K. 1996, A\&A, 309, 849

van Loon, J. Th. 2000, A\&A, 354, 125

van Loon, J. Th., Zijlstra, A. A., Whitelock, P. A., et al. 1998, A\&A, 329, 169 van Loon, J. Th., Zijlstra, A. A., \& Groenewegen, M. A. T. 1999, A\&A, 346, 805

Vanhollebeke, E., Groenewegen, M. A. T., \& Girardi, L. 2007, A\&A, submitted Vassiliadis, E., \& Wood, P. R. 1993, ApJ, 413, 641

Vázquez, G. A., \& Leitherer, C. 2005, ApJ, 621, 695

Ventura, P., D’Antona, F., \& Mazzitelli, I. 2002, A\&A, 393, 215

Verhoelst, T., Decin, L., van Malderen, R., et al. 2006, A\&A, 447, 311

Yan, H., Burstein, D., Fan, X., et al. 2000, PASP, 112, 691

Wachter, A., Schröder, K.-P., Winters, J. M., Arndt, T. U., \& Sedlmayr, E. 2002, A\&A, 384, 452

Wagenhuber, J. 1996, Ph.D. Thesis, Technische Universität München

Wagenhuber, J., \& Groenewegen, M. A. T. 1998, A\&A, 340, 183

Winters, J. M., Le Bertre, T., Jeong, K. S., Helling, Ch., \& Sedlmayr, E. 2000, A\&A, 361, 641

Winters, J. M., Le Bertre, T., Jeong, K. S., Nyman, L.-A., \& Epchtein, N. 2003, A\&A, 409, 715

Woitke, P. 2006, A\&A, 460, L9

Wood, P. R., Bessell, M. S., \& Fox, M. W. 1983, ApJ, 272, 99

Zijlstra, A. A., Matsuura, M., Wood, P. R., et al. 2006, MNRAS, 370, 1961 